CRYSTALLOGRAPHIC

COMMUNICATIONS

ISSN 2056-9890

Received 6 November 2014

Accepted 19 November 2014

Edited by H. Stoeckli-Evans, University of Neuchâtel, Switzerland

Keywords: crystal structure; cyclohexene; cyclcohexen-1-one

CCDC reference: 1035112

Supporting information: this article has supporting information at journals.iucr.org/e

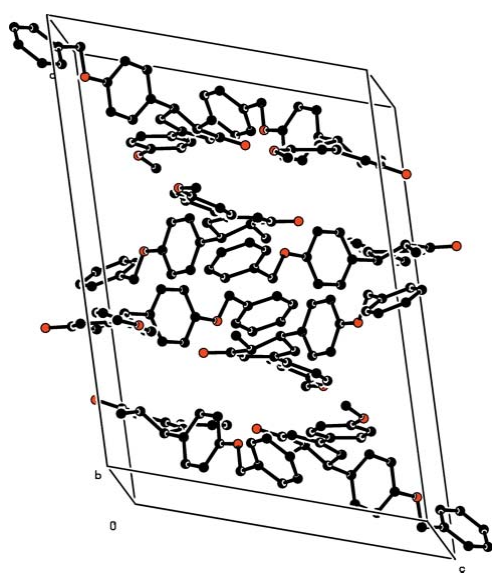

OPEN $\odot$ ACCESS

\section{Crystal structure of 5-(4-benzyloxyphenyl)-3-(4- methoxyphenyl)-6-methylcyclohex-2-en-1-one}

\author{
S. Sathya, ${ }^{a}$ D. Reuben Jonathan, ${ }^{b}$ J. Sidharthan, ${ }^{c}$ R. Vasanthi ${ }^{a}$ and G. Usha ${ }^{a *}$

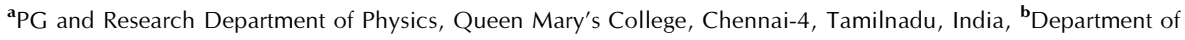 \\ Chemistry, Madras Christian College, Chennai-59, Tamilnadu, India, and 'PG \& Research Department of Chemistry, V. O. \\ Chidambaram College, Thoothukudi - 628 008, Tamil Nadu, India. *Correspondence e-mail: guqmc@yahoo.com
}

The title compound, $\mathrm{C}_{27} \mathrm{H}_{26} \mathrm{O}_{3}$, crystallized with two independent molecules ( $A$ and $B)$ in the asymmetric unit. In molecule $A$, the plane of the central benzene ring forms dihedral angles of $75.78(14)$ and $52.75(16)^{\circ}$ with that of the terminal benzene rings, and the dihedral angle between the planes of the terminal benzene rings is $51.49(17)^{\circ}$. The corresponding values for molecule $B$ are 75.18 (14), $58.11(16)$ and $47.91(16)^{\circ}$, respectively. The cyclohexene ring adopts an envelope conformation in both molecules, with the $\mathrm{C}$ atom to which is attached the central benzene ring as the flap. The crystal packing, is stabilized by $\mathrm{C}-\mathrm{H} \cdots \pi$ interactions.

\section{Structural commentary}

Cyclohexenone is a versatile intermediate used in the synthesis of a variety of chemical products such as pharmaceuticals and fragrances. Cyclohexenone and cyclohexenone derivatives are known for anti-inflammatory and analgesic activities (Kalluraya \& Rahiman, 2003). $\alpha, \beta$-Unsaturated carbonyl compounds have shown various biological activities such as antioxidant (Suksamrarn et al., 2003), antitumor (Kumar et al., 2003), anticancer (Modzelewska et al., 2006) and antimalarial (Ferrer et al., 2009). In addition, chalcones are widely used in cosmetic compositions (Forestier et al., 1989; Podraze, 1991) and in applications of dyes (Asiri, 2003). Cyclohexenone derivatives are well known lead molecules for the treatment of inflammation and autoimmune diseases (Tanaka et al., 1997). Apart from being biologically important compounds, chalcone derivatives show nonlinear optical (NLO) properties with excellent blue light transmittance and good crystallizability (Shettigar et al., 2006). In this context, herein we report the synthesis and crystal structure of the title compound.

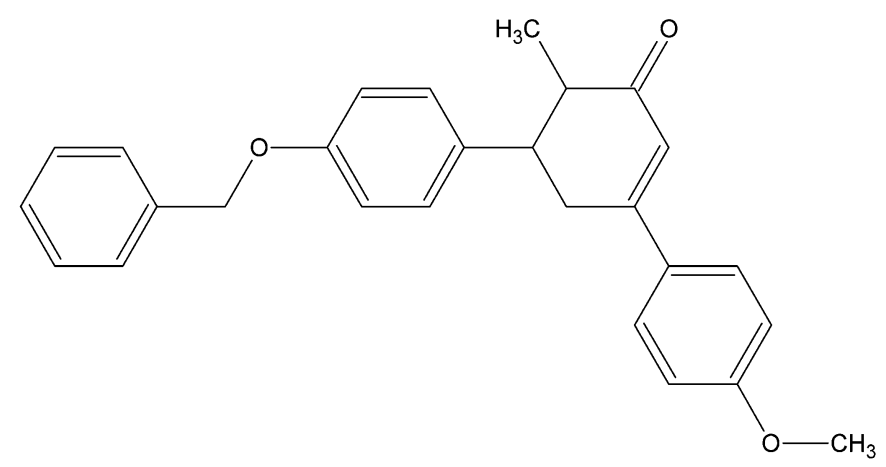

The title compound crystallized with two molecules ( $A$ and $B)$ in the asymmetric unit (Fig. 1). The benzyloxyphenyl and 


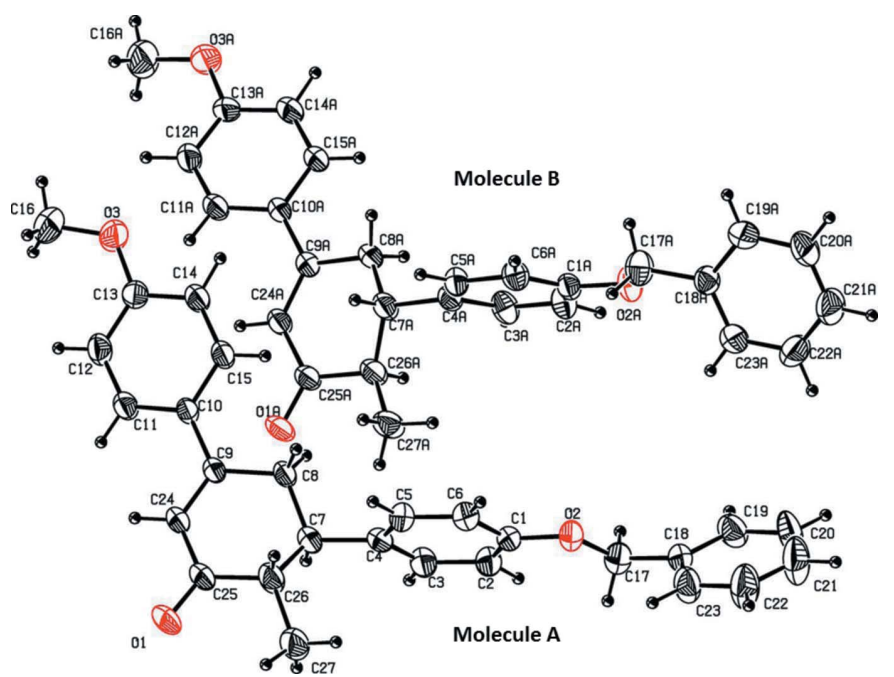

Figure 1

The molecular structure of the two independent molecules of the title compound, showing the atom labelling. Displacement ellipsoids are drawn at the $30 \%$ probability level.

the methoxyphenyl rings are linked with a cyclohexene ring. The $\mathrm{C} 25-\mathrm{O} 1$ and $\mathrm{C} 25 A-\mathrm{O} 1 A$ bond lengths of 1.228 (3) and 1.224 (3) A, respectively, indicate double-bond character. In both molecules, the $\mathrm{C}-\mathrm{O}$ bond lengths are in the range 1.362 (3)-1.414 (4) $\AA$ and represent single-bond character. In molecule $A$, the torsion angles $\mathrm{C} 5-\mathrm{C} 4-\mathrm{C} 7-\mathrm{C} 8=69.2(4)^{\circ}$ and $\mathrm{C} 24-\mathrm{C} 9-\mathrm{C} 10-\mathrm{C} 11=-16.0(4)^{\circ}$ show that the benzyloxyphenyl and methoxyphenyl groups have a $+s c$ and $-s p$ orientation with respect to the cyclohexene moiety. The

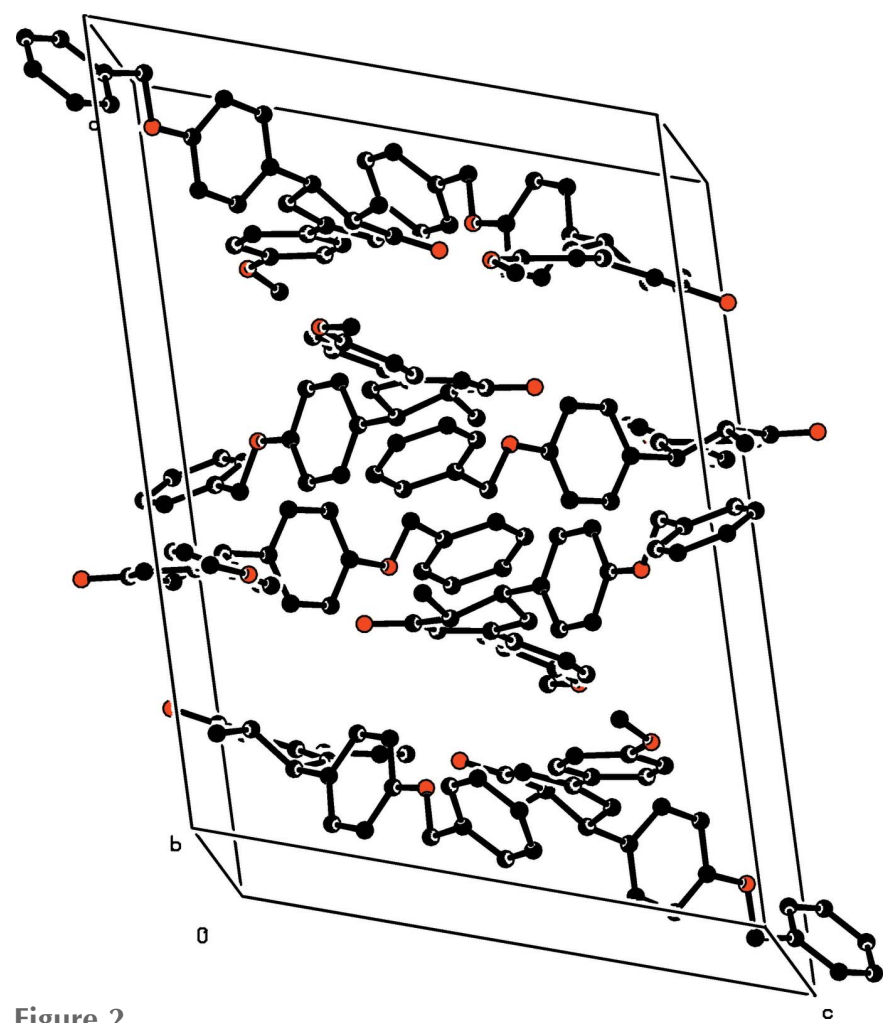

A view along the $b$ axis of the crystal packing of the title compound.
Table 1

Hydrogen-bond geometry $\left(\AA,^{\circ}\right)$.

$C g 3$ and $C g 8$ are the centroids of rings $\mathrm{C} 10-\mathrm{C} 15$ and $\mathrm{C} 10 A-\mathrm{C} 15 A$, respectively.

\begin{tabular}{lllll}
\hline$D-\mathrm{H} \cdots A$ & $D-\mathrm{H}$ & $\mathrm{H} \cdots A$ & $D \cdots A$ & $D-\mathrm{H} \cdots A$ \\
\hline $\mathrm{C} 2 A-\mathrm{H} 2 A \cdots C g 3^{\mathrm{i}}$ & 0.93 & 2.87 & $3.707(3)$ & 150 \\
$\mathrm{C} 16 A-\mathrm{H} 16 E \cdots C g 8^{\mathrm{ii}}$ & 0.96 & 2.92 & $3.866(5)$ & 168
\end{tabular}

Symmetry codes: (i) $x,-y+\frac{3}{2}, z+\frac{1}{2}$; (ii) $x,-y+\frac{3}{2}, z-\frac{1}{2}$.

arrangement in molecule $B$ is slightly different, with torsion angles $\mathrm{C} 5 A-\mathrm{C} 4 A-\mathrm{C} 7 A-\mathrm{C} 8 A=111.5(3)^{\circ}$ and $\mathrm{C} 24 A-$ $\mathrm{C} 9 A-\mathrm{C} 10 A-\mathrm{C} 11 A=20.8(4)^{\circ}$. The cyclohexene ring in both molecules adopts an envelope conformation with atoms $\mathrm{C} 7$ and $\mathrm{C} 7 A$ as the flaps in molecules $A$ and $B$, respectively.

The crystal packing (Fig. 2) is stabilized by $\mathrm{C}-\mathrm{H} \cdots \pi$ interactions (Table 1).

\section{Synthesis and crystallization}

(2E)-3-(4-Benzyloxyphenyl)-1-(4-methoxyphenyl)prop-2-en1-one was synthesized following the literature procedure of Ezhilarasi et al. (2014). The synthesis of the title compound was carried out by following the reported procedure of Fun et al. (2012). In a $100 \mathrm{ml}$ round-bottomeded flask, (2E)-3(4-benzyloxyphenyl)-1-(4-methoxyphenyl)prop-2-en-1-one $(0.01 \mathrm{~mol})$ and ethyl methyl ketone $(0.01 \mathrm{~mol})$ were refluxed in absolute alcohol $(50 \mathrm{ml})$ in the presence of $10 \%$ sodium hydroxide solution $(10 \mathrm{ml})$ for $1 \mathrm{~h}$ in an oil bath. The reaction

Table 2

Experimental details.

\begin{tabular}{|c|c|}
\hline Crystal data & \\
\hline Chemical formula & $\mathrm{C}_{27} \mathrm{H}_{26} \mathrm{O}_{3}$ \\
\hline$M_{\mathrm{r}}$ & 398.48 \\
\hline Crystal system, space group & Monoclinic, $P 2_{1} / c$ \\
\hline Temperature (K) & 293 \\
\hline$a, b, c(\AA)$ & $\begin{array}{l}20.5663(12), 15.2878(9) \\
\quad 14.5689(8)\end{array}$ \\
\hline$\beta\left(^{\circ}\right)$ & $107.938(4)$ \\
\hline$V\left(\AA^{3}\right)$ & $4358.0(4)$ \\
\hline$Z$ & 8 \\
\hline Radiation type & Мo $K \alpha$ \\
\hline$\mu\left(\mathrm{mm}^{-1}\right)$ & 0.08 \\
\hline Crystal size $(\mathrm{mm})$ & $0.25 \times 0.23 \times 0.20$ \\
\hline Data collection & \\
\hline Diffractometer & Bruker Kappa APEXII CCD \\
\hline Absorption correction & $\begin{array}{l}\text { Multi-scan (SADABS; Bruker, } \\
\text { 2008) }\end{array}$ \\
\hline$T_{\min }, T_{\max }$ & $0.981,0.985$ \\
\hline $\begin{array}{l}\text { No. of measured, independent and } \\
\text { observed }[I>2 \sigma(I)] \text { reflections }\end{array}$ & $33189,7901,3714$ \\
\hline$R_{\text {int }}$ & 0.057 \\
\hline$(\sin \theta / \lambda)_{\max }\left(\AA^{-1}\right)$ & 0.600 \\
\hline Refinement & \\
\hline$R\left[F^{2}>2 \sigma\left(F^{2}\right)\right], w R\left(F^{2}\right), S$ & $0.063,0.193,0.99$ \\
\hline No. of reflections & 7901 \\
\hline No. of parameters & 546 \\
\hline $\mathrm{H}$-atom treatment & $\mathrm{H}$-atom parameters constrained \\
\hline$\Delta \rho_{\max }, \Delta \rho_{\min }\left(\mathrm{e} \AA^{-3}\right)$ & $0.31,-0.21$ \\
\hline
\end{tabular}

Computer programs: APEX2 and SAINT (Bruker, 2008), SHELXS97 and SHELXL2014 (Sheldrick, 2008), ORTEP-3 for Windows (Farrugia, 2012), and PLATON (Spek, 2009). 
mixture was then cooled and the precipitate obtained filtered, washed with distilled water and dried. The crude product was recrystallized twice from absolute alcohol (yield 80\%), giving yellow block-like crystals.

\section{Refinement}

Crystal data, data collection and structure refinement details are summarized in Table 2. The $\mathrm{H}$ atoms were positioned geometrically and treated as riding atoms, with $\mathrm{C}-\mathrm{H}=0.93-$ $0.98 \AA$, and with $U_{\text {iso }}(\mathrm{H})=1.5 U_{\text {eq }}(\mathrm{C})$ for methyl $\mathrm{H}$ atoms and $1.2 U_{\text {eq }}(\mathrm{C})$ for other $\mathrm{H}$ atoms.

\section{Acknowledgements}

The authors thank Professor D. Velmurugan, Centre for Advanced Study in Crystallography and Biophysics, University of Madras, for providing data-collection facilities.

\section{References}

Asiri, A. M. (2003). Bull. Korean Chem. Soc. 24, 426-430.
Bruker (2008). APEX2, SAINT and SADABS. Bruker AXS Inc., Madison, Wisconsin, USA.

Ezhilarasi, K. S., Reuben Jonathan, D., Sathya, S., Prathebha, K. \& Usha, G. (2014). Acta Cryst. E70, o608-o609.

Farrugia, L. J. (2012). J. Appl. Cryst. 45, 849-854.

Ferrer, R., Lobo, G., Gamboa, N., Rodrigues, J., Abramjuk, C., Jung, K., Lein, M. \& Charris, J. E. (2009). Sci. Pharm. 77, 725-741.

Forestier, S., Moire, C. \& Lang, G. (1989). US Patent No. 4867964.

Fun, H.-K., Farhadikoutenaei, A., Sarojini, B. K., Mohan, B. J. \& Narayana, B. (2012). Acta Cryst. E68, o2788-o2789.

Kalluraya, B. \& Rahiman, M. A. (2003). Indian J. Chem. Sect. B, 42 , 1141-1148.

Kumar, S. K., Hager, E., Catherine, P., Gurulingappa, H., Davidson, N. E. \& Khan, S. R. (2003). J. Med. Chem. 46, 2813-2815.

Modzelewska, A., Pettit, C., Achanta, G., Davidson, N. E., Huang, P. \& Khan, S. R. (2006). Bioorg. Med. Chem. 14, 3491-3495.

Podraze, K. F. (1991). Org. Prep. Proced. Int. 23, 217.

Sheldrick, G. M. (2008). Acta Cryst. A64, 112-122.

Shettigar, S., Chandrasekharan, K., Umesh, G., Sarojini, B. K. \& Narayana, B. (2006). Polymer, 47, 3565-3567.

Spek, A. L. (2009). Acta Cryst. D65, 148-155.

Suksamrarn, A., Poomsing, P., Aroonrerk, N., Punjanon, T., Suksamrarn, S. \& Kongkun, S. (2003). Arch. Pharm. Res. 26, 816820.

Tanaka, M., Nara, F., Suzuki, K., Hosoya, T. \& Ogita, T. (1997). J. Am. Chem. Soc. 119, 7871-7872. 


\section{supporting information}

Acta Cryst. (2015). E71, 16-18 [https://doi.org/10.1107/S2056989014025390]

Crystal structure of 5-(4-benzyloxyphenyl)-3-(4-methoxyphenyl)-6-methylcyclohex-2-en-1-one

S. Sathya, D. Reuben Jonathan, J. Sidharthan, R. Vasanthi and G. Usha

Computing details

Data collection: APEX2 (Bruker, 2008); cell refinement: SAINT (Bruker, 2008); data reduction: SAINT (Bruker, 2008); program(s) used to solve structure: SHELXS97 (Sheldrick, 2008); program(s) used to refine structure: SHELXL2014 (Sheldrick, 2008); molecular graphics: ORTEP-3 for Windows (Farrugia, 2012); software used to prepare material for publication: SHELXL2014 (Sheldrick, 2008) and PLATON (Spek, 2009).

5-(4-Benzyloxyphenyl)-3-(4-methoxyphenyl)-6-methylcyclohex-2-en-1-one

Crystal data

$\mathrm{C}_{27} \mathrm{H}_{26} \mathrm{O}_{3}$

$M_{r}=398.48$

Monoclinic, $P 2_{1} / c$

$a=20.5663$ (12) $\AA$

$b=15.2878(9) \AA$

$c=14.5689(8) \AA$

$\beta=107.938(4)^{\circ}$

$V=4358.0(4) \AA^{3}$

$Z=8$

\section{Data collection}

Bruker Kappa APEXII CCD diffractometer

Radiation source: fine-focus sealed tube Graphite monochromator $\omega$ and $\varphi$ scan

Absorption correction: multi-scan

(SADABS; Bruker, 2008)

$T_{\min }=0.981, T_{\max }=0.985$

\section{Refinement}

Refinement on $F^{2}$

Least-squares matrix: full

$R\left[F^{2}>2 \sigma\left(F^{2}\right)\right]=0.063$

$w R\left(F^{2}\right)=0.193$

$S=0.99$

7901 reflections

546 parameters

0 restraints

Hydrogen site location: inferred from neighbouring sites
$F(000)=1696$

$D_{\mathrm{x}}=1.215 \mathrm{Mg} \mathrm{m}^{-3}$

Mo $K \alpha$ radiation, $\lambda=0.71073 \AA$

Cell parameters from 10904 reflections

$\theta=1.0-28.4^{\circ}$

$\mu=0.08 \mathrm{~mm}^{-1}$

$T=293 \mathrm{~K}$

Block, yellow

$0.25 \times 0.23 \times 0.20 \mathrm{~mm}$

33189 measured reflections

7901 independent reflections

3714 reflections with $I>2 \sigma(I)$

$R_{\text {int }}=0.057$

$\theta_{\max }=25.3^{\circ}, \theta_{\min }=1.0^{\circ}$

$h=-24 \rightarrow 24$

$k=-18 \rightarrow 14$

$l=-17 \rightarrow 17$

$\mathrm{H}$-atom parameters constrained $w=1 /\left[\sigma^{2}\left(F_{\mathrm{o}}^{2}\right)+(0.1028 P)^{2}\right]$ where $P=\left(F_{\mathrm{o}}^{2}+2 F_{\mathrm{c}}^{2}\right) / 3$

$(\Delta / \sigma)_{\max }<0.001$

$\Delta \rho_{\max }=0.31$ e $\AA^{-3}$

$\Delta \rho_{\text {min }}=-0.21$ e $\AA^{-3}$

Extinction correction: SHELXL2014

(Sheldrick, 2008),

$\mathrm{Fc}^{*}=\mathrm{kFc}\left[1+0.001 \times \mathrm{Fc}^{2} \lambda^{3} / \sin (2 \theta)\right]^{-1 / 4}$

Extinction coefficient: 0.0020 (5) 


\section{Special details}

Geometry. All esds (except the esd in the dihedral angle between two 1.s. planes) are estimated using the full covariance matrix. The cell esds are taken into account individually in the estimation of esds in distances, angles and torsion angles; correlations between esds in cell parameters are only used when they are defined by crystal symmetry. An approximate (isotropic) treatment of cell esds is used for estimating esds involving l.s. planes.

Fractional atomic coordinates and isotropic or equivalent isotropic displacement parameters $\left(\AA^{2}\right)$

\begin{tabular}{|c|c|c|c|c|}
\hline & $x$ & $y$ & $z$ & $U_{\text {iso }} * / U_{\text {eq }}$ \\
\hline $\mathrm{O} 1$ & $0.16345(12)$ & $0.76886(14)$ & $-0.02540(14)$ & $0.0871(7)$ \\
\hline $\mathrm{O} 2$ & $0.09475(10)$ & $1.08899(13)$ & $0.43402(13)$ & $0.0703(6)$ \\
\hline $\mathrm{O} 3$ & $0.19511(12)$ & $0.32014(14)$ & $0.35402(16)$ & $0.0890(7)$ \\
\hline $\mathrm{C} 1$ & $0.09377(16)$ & $1.02145(18)$ & $0.37052(19)$ & $0.0568(7)$ \\
\hline $\mathrm{C} 2$ & $0.03609(16)$ & $0.9860(2)$ & $0.3070(2)$ & $0.0666(8)$ \\
\hline $\mathrm{H} 2$ & -0.0069 & 1.0070 & 0.3045 & $0.080^{*}$ \\
\hline $\mathrm{C} 3$ & $0.04228(17)$ & $0.91803(19)$ & $0.2459(2)$ & $0.0685(8)$ \\
\hline $\mathrm{H} 3$ & 0.0030 & 0.8946 & 0.2026 & $0.082 *$ \\
\hline $\mathrm{C} 4$ & $0.10415(17)$ & $0.88533(19)$ & $0.24821(19)$ & $0.0611(8)$ \\
\hline $\mathrm{C} 5$ & $0.16111(17)$ & $0.9217(2)$ & $0.3127(2)$ & $0.0725(9)$ \\
\hline H5 & 0.2039 & 0.9000 & 0.3156 & $0.087^{*}$ \\
\hline C6 & $0.15690(17)$ & $0.9899(2)$ & $0.3739(2)$ & $0.0680(8)$ \\
\hline H6 & 0.1963 & 1.0138 & 0.4165 & $0.082 *$ \\
\hline $\mathrm{C} 7$ & $0.11115(18)$ & $0.81052(19)$ & $0.18306(19)$ & $0.0728(9)$ \\
\hline $\mathrm{H} 7$ & 0.0646 & 0.7984 & 0.1416 & $0.087^{*}$ \\
\hline $\mathrm{C} 8$ & $0.13470(14)$ & $0.72774(17)$ & $0.23821(17)$ & $0.0540(7)$ \\
\hline H8A & 0.1003 & 0.7096 & 0.2673 & $0.065^{*}$ \\
\hline H8B & 0.1763 & 0.7400 & 0.2901 & $0.065^{*}$ \\
\hline C9 & $0.14816(13)$ & $0.65290(17)$ & $0.17931(16)$ & $0.0468(7)$ \\
\hline $\mathrm{C} 10$ & $0.15636(12)$ & $0.56499(17)$ & $0.22096(17)$ & $0.0471(7)$ \\
\hline $\mathrm{C} 11$ & $0.15574(14)$ & $0.48994(19)$ & $0.16688(19)$ & $0.0598(8)$ \\
\hline H11 & 0.1469 & 0.4953 & 0.1006 & $0.072^{*}$ \\
\hline $\mathrm{C} 12$ & $0.16766(15)$ & $0.4084(2)$ & $0.2074(2)$ & $0.0664(8)$ \\
\hline H12 & 0.1670 & 0.3599 & 0.1687 & $0.080^{*}$ \\
\hline $\mathrm{C} 13$ & $0.18067(14)$ & $0.39773(19)$ & $0.3056(2)$ & $0.0603(8)$ \\
\hline C14 & $0.17958(16)$ & $0.4705(2)$ & $0.3607(2)$ & $0.0687(9)$ \\
\hline H14 & 0.1870 & 0.4641 & 0.4266 & $0.082 *$ \\
\hline $\mathrm{C} 15$ & $0.16775(15)$ & $0.55200(19)$ & $0.32023(19)$ & $0.0602(8)$ \\
\hline H15 & 0.1672 & 0.5999 & 0.3593 & $0.072 *$ \\
\hline $\mathrm{C} 16$ & $0.2115(3)$ & $0.2467(2)$ & $0.3082(3)$ & $0.1329(17)$ \\
\hline H16A & 0.2251 & 0.1997 & 0.3538 & $0.199^{*}$ \\
\hline H16B & 0.2484 & 0.2609 & 0.2834 & $0.199^{*}$ \\
\hline $\mathrm{H} 16 \mathrm{C}$ & 0.1723 & 0.2293 & 0.2561 & $0.199^{*}$ \\
\hline $\mathrm{C} 17$ & $0.03159(16)$ & $1.1250(2)$ & $0.4337(2)$ & $0.0766(9)$ \\
\hline H17A & 0.0054 & 1.0821 & 0.4564 & $0.092 *$ \\
\hline H17B & 0.0054 & 1.1422 & 0.3688 & $0.092 *$ \\
\hline $\mathrm{C} 18$ & $0.04583(16)$ & $1.2034(2)$ & $0.4992(2)$ & $0.0624(8)$ \\
\hline C19 & $0.01360(17)$ & $1.2129(2)$ & $0.5687(2)$ & $0.0813(10)$ \\
\hline H19 & -0.0156 & 1.1695 & 0.5775 & $0.098^{*}$ \\
\hline
\end{tabular}




\begin{tabular}{|c|c|c|c|c|}
\hline $\mathrm{C} 20$ & $0.0249(2)$ & $1.2872(3)$ & $0.6252(3)$ & $0.1090(13)$ \\
\hline $\mathrm{H} 20$ & 0.0027 & 1.2936 & 0.6716 & $0.131 *$ \\
\hline $\mathrm{C} 21$ & $0.0673(2)$ & $1.3505(3)$ & $0.6144(3)$ & $0.1217(16)$ \\
\hline $\mathrm{H} 21$ & 0.0743 & 1.4004 & 0.6528 & $0.146^{*}$ \\
\hline $\mathrm{C} 22$ & $0.0995(2)$ & $1.3410(2)$ & $0.5475(3)$ & $0.1185(15)$ \\
\hline $\mathrm{H} 22$ & 0.1292 & 1.3844 & 0.5400 & $0.142 *$ \\
\hline $\mathrm{C} 23$ & 0.08895 (19) & $1.2682(2)$ & $0.4904(3)$ & $0.0904(11)$ \\
\hline $\mathrm{H} 23$ & 0.1117 & 1.2627 & 0.4445 & $0.108^{*}$ \\
\hline $\mathrm{C} 24$ & $0.15546(14)$ & 0.66994 (19) & $0.09195(18)$ & $0.0576(8)$ \\
\hline $\mathrm{H} 24$ & 0.1613 & 0.6230 & 0.0547 & $0.069^{*}$ \\
\hline $\mathrm{C} 25$ & $0.15466(15)$ & $0.7571(2)$ & $0.05331(19)$ & $0.0642(8)$ \\
\hline $\mathrm{C} 26$ & $0.1498(2)$ & $0.8335(2)$ & $0.1167(2)$ & $0.0865(11)$ \\
\hline $\mathrm{H} 26$ & 0.1968 & 0.8428 & 0.1585 & $0.104 *$ \\
\hline $\mathrm{C} 27$ & $0.1309(2)$ & $0.9172(2)$ & $0.0609(3)$ & $0.1127(14)$ \\
\hline H27A & 0.1608 & 0.9264 & 0.0226 & $0.169^{*}$ \\
\hline H27B & 0.1351 & 0.9651 & 0.1050 & $0.169^{*}$ \\
\hline $\mathrm{H} 27 \mathrm{C}$ & 0.0845 & 0.9136 & 0.0195 & $0.169^{*}$ \\
\hline O1A & $0.31086(10)$ & $0.78628(13)$ & $0.34094(12)$ & $0.0725(6)$ \\
\hline $\mathrm{O} 2 \mathrm{~A}$ & $0.41660(10)$ & $1.09227(13)$ & $0.87045(14)$ & $0.0687(6)$ \\
\hline $\mathrm{O} 3 \mathrm{~A}$ & $0.31787(13)$ & $0.31427(14)$ & $0.67545(16)$ & $0.0924(8)$ \\
\hline $\mathrm{C} 1 \mathrm{~A}$ & $0.41218(15)$ & $1.02388(17)$ & $0.80701(18)$ & $0.0520(7)$ \\
\hline $\mathrm{C} 2 \mathrm{~A}$ & $0.34719(15)$ & 0.99367 (19) & $0.7637(2)$ & $0.0640(8)$ \\
\hline $\mathrm{H} 2 \mathrm{~A}$ & 0.3105 & 1.0188 & 0.7786 & $0.077^{*}$ \\
\hline $\mathrm{C} 3 \mathrm{~A}$ & $0.33616(15)$ & $0.9262(2)$ & $0.6981(2)$ & $0.0671(8)$ \\
\hline $\mathrm{H} 3 \mathrm{~A}$ & 0.2920 & 0.9055 & 0.6701 & $0.080^{*}$ \\
\hline $\mathrm{C} 4 \mathrm{~A}$ & $0.38969(15)$ & $0.88854(17)$ & $0.67276(18)$ & $0.0536(7)$ \\
\hline $\mathrm{C} 5 \mathrm{~A}$ & $0.45373(15)$ & $0.91928(18)$ & $0.71752(19)$ & $0.0581(8)$ \\
\hline $\mathrm{H} 5 \mathrm{~A}$ & 0.4905 & 0.8946 & 0.7023 & $0.070^{*}$ \\
\hline C6A & $0.46610(15)$ & 0.98615 (19) & $0.78505(19)$ & $0.0610(8)$ \\
\hline H6A & 0.5105 & 1.0052 & 0.8152 & $0.073^{*}$ \\
\hline C7A & $0.37676(15)$ & $0.81644(18)$ & $0.59723(18)$ & $0.0614(8)$ \\
\hline $\mathrm{H} 7 \mathrm{~A}$ & 0.4214 & 0.8040 & 0.5892 & $0.074^{*}$ \\
\hline $\mathrm{C} 8 \mathrm{~A}$ & $0.35410(14)$ & $0.73180(17)$ & $0.63033(17)$ & $0.0563(7)$ \\
\hline H8A1 & 0.3895 & 0.7118 & 0.6873 & $0.068^{*}$ \\
\hline H8A2 & 0.3133 & 0.7427 & 0.6487 & $0.068^{*}$ \\
\hline C9A & $0.33916(13)$ & $0.66057(17)$ & $0.55616(17)$ & $0.0502(7)$ \\
\hline $\mathrm{C} 10 \mathrm{~A}$ & $0.33737(13)$ & $0.56976(18)$ & $0.58785(17)$ & $0.0501(7)$ \\
\hline $\mathrm{C} 11 \mathrm{~A}$ & $0.34401(14)$ & $0.4993(2)$ & $0.53198(18)$ & $0.0612(8)$ \\
\hline H11A & 0.3521 & 0.5102 & 0.4736 & $0.073^{*}$ \\
\hline $\mathrm{C} 12 \mathrm{~A}$ & $0.33929(15)$ & $0.4141(2)$ & $0.5583(2)$ & $0.0653(8)$ \\
\hline H12A & 0.3453 & 0.3687 & 0.5192 & $0.078^{*}$ \\
\hline $\mathrm{C} 13 \mathrm{~A}$ & $0.32555(15)$ & 0.39603 (19) & $0.6431(2)$ & $0.0637(8)$ \\
\hline $\mathrm{C} 14 \mathrm{~A}$ & $0.31987(18)$ & $0.4637(2)$ & $0.7010(2)$ & $0.0805(10)$ \\
\hline H14A & 0.3116 & 0.4518 & 0.7590 & $0.097^{*}$ \\
\hline $\mathrm{C} 15 \mathrm{~A}$ & $0.32618(15)$ & $0.5493(2)$ & $0.67559(19)$ & $0.0678(9)$ \\
\hline $\mathrm{H} 15 \mathrm{~A}$ & 0.3230 & 0.5941 & 0.7171 & $0.081^{*}$ \\
\hline $\mathrm{C} 16 \mathrm{~A}$ & $0.3163(2)$ & $0.2418(2)$ & $0.6145(3)$ & $0.1197(15)$ \\
\hline H16D & 0.3133 & 0.1888 & 0.6482 & $0.180^{*}$ \\
\hline
\end{tabular}




$\begin{array}{lllll}\text { H16E } & 0.3572 & 0.2410 & 0.5960 & 0.180^{*} \\ \text { H16F } & 0.2772 & 0.2465 & 0.5579 & 0.180^{*} \\ \text { C17A } & 0.48132(16) & 1.1279(2) & 0.9177(2) & 0.0749(9) \\ \text { H17C } & 0.5054 & 1.1398 & 0.8713 & 0.090^{*} \\ \text { H17D } & 0.5082 & 1.0866 & 0.9648 & 0.090^{*} \\ \text { C18A } & 0.47207(14) & 1.21032(18) & 0.9665(2) & 0.0574(8) \\ \text { C19A } & 0.50644(16) & 1.2251(2) & 1.0626(2) & 0.0757(9) \\ \text { H19A } & 0.5340 & 1.1816 & 1.0994 & 0.091^{*} \\ \text { C20A } & 0.5000(2) & 1.3042(3) & 1.1042(2) & 0.0982(12) \\ \text { H20A } & 0.5234 & 1.3133 & 1.1691 & 0.118^{*} \\ \text { C21A } & 0.4603(2) & 1.3691(3) & 1.0527(3) & 0.0980(12) \\ \text { H21A } & 0.4567 & 1.4224 & 1.0815 & 0.118^{*} \\ \text { C22A } & 0.42632(18) & 1.3549(2) & 0.9592(3) & 0.0905(11) \\ \text { H22A } & 0.3984 & 1.3987 & 0.9234 & 0.109^{*} \\ \text { C23A } & 0.43207(16) & 1.2772(2) & 0.9156(2) & 0.0741(9) \\ \text { H23A } & 0.4085 & 1.2695 & 0.8505 & 0.089^{*} \\ \text { C24A } & 0.32630(14) & 0.68225(18) & 0.46206(18) & 0.0550(7) \\ \text { H24A } & 0.3189 & 0.6372 & 0.4171 & 0.066^{*} \\ \text { C25A } & 0.32344(14) & 0.77137(19) & 0.42723(18) & 0.0560(7) \\ \text { C26A } & 0.33199(16) & 0.84476(18) & 0.49948(18) & 0.0660(8) \\ \text { H26A } & 0.2866 & 0.8546 & 0.5062 & 0.079^{*} \\ \text { C27A } & 0.35178(17) & 0.92969(18) & 0.4628(2) & 0.0783(10) \\ \text { H27D } & 0.3242 & 0.9385 & 0.3971 & 0.117^{*} \\ \text { H27E } & 0.3991 & 0.9276 & 0.4662 & 0.117^{*} \\ \text { H27F } & 0.3446 & 0.9771 & 0.5018 & 0.117^{*}\end{array}$

Atomic displacement parameters $\left(\AA^{2}\right)$

\begin{tabular}{lllllll}
\hline & $U^{11}$ & $U^{22}$ & $U^{33}$ & $U^{12}$ & $U^{13}$ & $U^{23}$ \\
\hline O1 & $0.137(2)$ & $0.0897(17)$ & $0.0539(12)$ & $0.0068(13)$ & $0.0580(13)$ & $0.0091(11)$ \\
O2 & $0.0811(15)$ & $0.0689(14)$ & $0.0623(12)$ & $0.0179(11)$ & $0.0242(10)$ & $-0.0115(11)$ \\
O3 & $0.142(2)$ & $0.0552(15)$ & $0.0934(16)$ & $0.0115(13)$ & $0.0706(15)$ & $0.0121(13)$ \\
C1 & $0.078(2)$ & $0.0490(19)$ & $0.0469(16)$ & $0.0108(16)$ & $0.0249(15)$ & $0.0037(15)$ \\
C2 & $0.077(2)$ & $0.064(2)$ & $0.0652(19)$ & $0.0079(17)$ & $0.0317(17)$ & $-0.0022(17)$ \\
C3 & $0.085(2)$ & $0.063(2)$ & $0.0616(18)$ & $0.0008(17)$ & $0.0284(16)$ & $-0.0073(17)$ \\
C4 & $0.089(2)$ & $0.0539(19)$ & $0.0407(15)$ & $0.0054(18)$ & $0.0209(16)$ & $0.0042(14)$ \\
C5 & $0.090(2)$ & $0.069(2)$ & $0.0664(19)$ & $0.0242(18)$ & $0.0344(18)$ & $0.0026(18)$ \\
C6 & $0.083(2)$ & $0.066(2)$ & $0.0554(17)$ & $0.0114(18)$ & $0.0213(16)$ & $-0.0032(16)$ \\
C7 & $0.120(3)$ & $0.061(2)$ & $0.0453(16)$ & $0.0132(18)$ & $0.0367(17)$ & $0.0002(15)$ \\
C8 & $0.0754(19)$ & $0.0525(19)$ & $0.0412(14)$ & $-0.0031(14)$ & $0.0284(13)$ & $-0.0042(14)$ \\
C9 & $0.0563(17)$ & $0.0478(18)$ & $0.0386(13)$ & $-0.0010(13)$ & $0.0182(12)$ & $-0.0064(13)$ \\
C10 & $0.0566(17)$ & $0.0483(18)$ & $0.0419(13)$ & $-0.0023(13)$ & $0.0231(12)$ & $-0.0076(14)$ \\
C11 & $0.078(2)$ & $0.058(2)$ & $0.0452(15)$ & $-0.0028(16)$ & $0.0214(14)$ & $-0.0085(16)$ \\
C12 & $0.085(2)$ & $0.057(2)$ & $0.0624(18)$ & $-0.0053(16)$ & $0.0308(16)$ & $-0.0175(17)$ \\
C13 & $0.081(2)$ & $0.0457(19)$ & $0.0661(18)$ & $-0.0003(15)$ & $0.0404(16)$ & $0.0031(17)$ \\
C14 & $0.108(3)$ & $0.059(2)$ & $0.0527(17)$ & $0.0027(17)$ & $0.0448(17)$ & $-0.0004(17)$ \\
C15 & $0.091(2)$ & $0.0497(19)$ & $0.0504(16)$ & $0.0001(15)$ & $0.0378(15)$ & $-0.0039(15)$ \\
C16 & $0.231(5)$ & $0.065(3)$ & $0.123(3)$ & $0.039(3)$ & $0.084(3)$ & $0.007(3)$
\end{tabular}




\begin{tabular}{|c|c|c|c|c|c|c|}
\hline $\mathrm{C} 17$ & $0.082(2)$ & $0.074(2)$ & $0.077(2)$ & $0.0138(18)$ & $0.0280(17)$ & $-0.0069(18)$ \\
\hline $\mathrm{C} 18$ & $0.081(2)$ & $0.058(2)$ & $0.0554(17)$ & $0.0123(17)$ & $0.0311(15)$ & $0.0003(16)$ \\
\hline $\mathrm{C} 19$ & $0.099(3)$ & $0.086(3)$ & $0.072(2)$ & $-0.008(2)$ & $0.0455(19)$ & $-0.002(2)$ \\
\hline $\mathrm{C} 20$ & $0.136(4)$ & $0.126(4)$ & $0.094(3)$ & $-0.013(3)$ & $0.078(3)$ & $-0.041(3)$ \\
\hline $\mathrm{C} 21$ & $0.169(4)$ & $0.085(3)$ & $0.143(4)$ & -0.024 (3) & $0.096(4)$ & $-0.049(3)$ \\
\hline $\mathrm{C} 22$ & $0.182(4)$ & $0.064(3)$ & $0.150(4)$ & $-0.022(3)$ & $0.109(3)$ & $-0.027(3)$ \\
\hline $\mathrm{C} 23$ & $0.133(3)$ & $0.071(3)$ & $0.096(3)$ & $-0.004(2)$ & $0.079(2)$ & $-0.005(2)$ \\
\hline $\mathrm{C} 24$ & $0.077(2)$ & $0.060(2)$ & $0.0412(14)$ & $0.0015(15)$ & $0.0271(13)$ & $-0.0041(14)$ \\
\hline $\mathrm{C} 25$ & $0.087(2)$ & $0.069(2)$ & $0.0443(15)$ & $0.0024(16)$ & $0.0328(15)$ & $0.0007(16)$ \\
\hline $\mathrm{C} 26$ & $0.156(3)$ & $0.057(2)$ & $0.072(2)$ & $-0.003(2)$ & $0.072(2)$ & $-0.0001(18)$ \\
\hline $\mathrm{C} 27$ & $0.215(5)$ & $0.065(2)$ & $0.087(2)$ & $0.015(3)$ & $0.088(3)$ & $0.014(2)$ \\
\hline O1A & $0.1121(17)$ & $0.0722(14)$ & $0.0350(10)$ & $-0.0027(11)$ & $0.0252(10)$ & $0.0048(10)$ \\
\hline $\mathrm{O} 2 \mathrm{~A}$ & $0.0722(14)$ & $0.0668(14)$ & $0.0740(13)$ & $-0.0165(11)$ & $0.0329(10)$ & $-0.0276(11)$ \\
\hline $\mathrm{O} 3 \mathrm{~A}$ & $0.160(2)$ & $0.0531(15)$ & $0.0759(15)$ & $0.0002(13)$ & $0.0538(15)$ & $0.0073(13)$ \\
\hline $\mathrm{C} 1 \mathrm{~A}$ & $0.072(2)$ & $0.0428(17)$ & $0.0463(15)$ & $-0.0049(15)$ & $0.0251(14)$ & $-0.0036(13)$ \\
\hline $\mathrm{C} 2 \mathrm{~A}$ & $0.061(2)$ & $0.074(2)$ & $0.0643(18)$ & $-0.0085(16)$ & $0.0289(15)$ & $-0.0163(17)$ \\
\hline $\mathrm{C} 3 \mathrm{~A}$ & $0.069(2)$ & $0.075(2)$ & $0.0588(17)$ & -0.0219 (17) & $0.0224(15)$ & $-0.0186(17)$ \\
\hline $\mathrm{C} 4 \mathrm{~A}$ & $0.073(2)$ & $0.0475(18)$ & $0.0463(15)$ & $-0.0014(16)$ & $0.0273(15)$ & $-0.0013(14)$ \\
\hline $\mathrm{C} 5 \mathrm{~A}$ & $0.067(2)$ & 0.0558 (19) & $0.0552(16)$ & $0.0015(15)$ & $0.0241(15)$ & $-0.0093(15)$ \\
\hline C6A & $0.0602(19)$ & $0.064(2)$ & $0.0586(17)$ & $-0.0049(16)$ & $0.0182(15)$ & $-0.0082(16)$ \\
\hline C7A & $0.085(2)$ & $0.056(2)$ & $0.0453(15)$ & $-0.0053(16)$ & $0.0231(14)$ & $-0.0045(14)$ \\
\hline $\mathrm{C} 8 \mathrm{~A}$ & $0.080(2)$ & $0.0552(19)$ & $0.0351(14)$ & $0.0022(15)$ & $0.0189(13)$ & $-0.0016(13)$ \\
\hline C9A & $0.0621(18)$ & $0.0490(18)$ & $0.0422(14)$ & $0.0017(13)$ & $0.0200(12)$ & $-0.0006(13)$ \\
\hline $\mathrm{C} 10 \mathrm{~A}$ & $0.0636(18)$ & $0.0516(19)$ & $0.0366(13)$ & $0.0010(14)$ & $0.0177(12)$ & $-0.0045(14)$ \\
\hline $\mathrm{C} 11 \mathrm{~A}$ & $0.086(2)$ & $0.059(2)$ & $0.0448(15)$ & $-0.0015(16)$ & $0.0300(15)$ & $0.0005(15)$ \\
\hline $\mathrm{C} 12 \mathrm{~A}$ & $0.094(2)$ & $0.051(2)$ & $0.0571(17)$ & $-0.0006(16)$ & $0.0315(15)$ & $-0.0081(15)$ \\
\hline $\mathrm{C} 13 \mathrm{~A}$ & $0.095(2)$ & $0.048(2)$ & 0.0507 (17) & $0.0035(16)$ & $0.0271(16)$ & $0.0075(16)$ \\
\hline $\mathrm{C} 14 \mathrm{~A}$ & $0.139(3)$ & $0.066(2)$ & 0.0494 (17) & $0.011(2)$ & $0.0480(19)$ & $0.0085(17)$ \\
\hline $\mathrm{C} 15 \mathrm{~A}$ & $0.108(3)$ & $0.058(2)$ & $0.0445(16)$ & $0.0095(17)$ & $0.0330(16)$ & $0.0047(15)$ \\
\hline $\mathrm{C} 16 \mathrm{~A}$ & $0.203(5)$ & $0.060(3)$ & $0.118(3)$ & $-0.006(3)$ & $0.082(3)$ & $-0.006(2)$ \\
\hline C17A & $0.075(2)$ & $0.066(2)$ & $0.083(2)$ & $-0.0095(18)$ & $0.0219(17)$ & $-0.0221(18)$ \\
\hline $\mathrm{C} 18 \mathrm{~A}$ & $0.069(2)$ & $0.0463(18)$ & $0.0576(17)$ & $-0.0063(15)$ & $0.0204(15)$ & $-0.0087(16)$ \\
\hline C19A & $0.087(2)$ & $0.077(3)$ & $0.0570(19)$ & $0.0107(18)$ & $0.0135(17)$ & $0.0019(18)$ \\
\hline $\mathrm{C} 20 \mathrm{~A}$ & $0.121(3)$ & $0.108(3)$ & 0.0535 (19) & $0.014(3)$ & $0.009(2)$ & $-0.028(2)$ \\
\hline $\mathrm{C} 21 \mathrm{~A}$ & $0.118(3)$ & 0.080 & $0.084(3)$ & $0.008(2)$ & $0.013(2)$ & $-0.032(2)$ \\
\hline $\mathrm{C} 22 \mathrm{~A}$ & $0.116(3)$ & $0.060(2)$ & $0.085(2)$ & $0.022(2)$ & $0.016(2)$ & $-0.006(2)$ \\
\hline $\mathrm{C} 23 \mathrm{~A}$ & $0.094(2)$ & $0.069(2)$ & $0.0540(18)$ & $0.0051(19)$ & $0.0150(17)$ & $-0.0060(18)$ \\
\hline $\mathrm{C} 24 \mathrm{~A}$ & $0.076(2)$ & 0.0519 (19) & 0.0397 (14) & $-0.0051(14)$ & $0.0214(13)$ & $-0.0058(14)$ \\
\hline $\mathrm{C} 25 \mathrm{~A}$ & $0.0711(19)$ & $0.059(2)$ & $0.0409(15)$ & $-0.0023(15)$ & $0.0217(13)$ & $0.0021(14)$ \\
\hline $\mathrm{C} 26 \mathrm{~A}$ & $0.099(2)$ & $0.058(2)$ & $0.0440(15)$ & $-0.0051(16)$ & $0.0271(15)$ & $-0.0009(15)$ \\
\hline $\mathrm{C} 27 \mathrm{~A}$ & $0.122(3)$ & $0.060(2)$ & 0.0555 (17) & $-0.0166(18)$ & $0.0302(18)$ & $0.0059(16)$ \\
\hline
\end{tabular}

Geometric parameters $\left(\AA,{ }^{\circ}\right)$

\begin{tabular}{llll}
\hline $\mathrm{O} 1-\mathrm{C} 25$ & $1.228(3)$ & $\mathrm{O} 1 \mathrm{~A}-\mathrm{C} 25 \mathrm{~A}$ & $1.224(3)$ \\
$\mathrm{O} 2-\mathrm{C} 1$ & $1.382(3)$ & $\mathrm{O} 2 \mathrm{~A}-\mathrm{C} 1 \mathrm{~A}$ & $1.380(3)$ \\
$\mathrm{O} 2-\mathrm{C} 17$ & $1.410(3)$ & $\mathrm{O} 2 \mathrm{~A}-\mathrm{C} 17 \mathrm{~A}$ & $1.406(3)$ \\
$\mathrm{O} 3-\mathrm{C} 13$ & $1.366(3)$ & $\mathrm{O} 3 \mathrm{~A}-\mathrm{C} 13 \mathrm{~A}$ & $1.362(3)$
\end{tabular}




\begin{tabular}{|c|c|c|c|}
\hline $\mathrm{O} 3-\mathrm{C} 16$ & $1.399(4)$ & $\mathrm{O} 3 \mathrm{~A}-\mathrm{C} 16 \mathrm{~A}$ & $1.414(4)$ \\
\hline $\mathrm{C} 1-\mathrm{C} 6$ & $1.371(4)$ & $\mathrm{C} 1 \mathrm{~A}-\mathrm{C} 2 \mathrm{~A}$ & $1.371(4)$ \\
\hline $\mathrm{C} 1-\mathrm{C} 2$ & $1.372(4)$ & $\mathrm{C} 1 \mathrm{~A}-\mathrm{C} 6 \mathrm{~A}$ & $1.372(4)$ \\
\hline $\mathrm{C} 2-\mathrm{C} 3$ & $1.399(4)$ & $\mathrm{C} 2 \mathrm{~A}-\mathrm{C} 3 \mathrm{~A}$ & $1.376(4)$ \\
\hline $\mathrm{C} 2-\mathrm{H} 2$ & 0.9300 & $\mathrm{C} 2 \mathrm{~A}-\mathrm{H} 2 \mathrm{~A}$ & 0.9300 \\
\hline $\mathrm{C} 3-\mathrm{C} 4$ & $1.358(4)$ & $\mathrm{C} 3 \mathrm{~A}-\mathrm{C} 4 \mathrm{~A}$ & $1.390(4)$ \\
\hline $\mathrm{C} 3-\mathrm{H} 3$ & 0.9300 & $\mathrm{C} 3 \mathrm{~A}-\mathrm{H} 3 \mathrm{~A}$ & 0.9300 \\
\hline $\mathrm{C} 4-\mathrm{C} 5$ & $1.373(4)$ & $\mathrm{C} 4 \mathrm{~A}-\mathrm{C} 5 \mathrm{~A}$ & $1.361(4)$ \\
\hline $\mathrm{C} 4-\mathrm{C} 7$ & $1.521(4)$ & $\mathrm{C} 4 \mathrm{~A}-\mathrm{C} 7 \mathrm{~A}$ & $1.522(4)$ \\
\hline $\mathrm{C} 5-\mathrm{C} 6$ & $1.392(4)$ & $\mathrm{C} 5 \mathrm{~A}-\mathrm{C} 6 \mathrm{~A}$ & $1.387(4)$ \\
\hline C5-H5 & 0.9300 & $\mathrm{C} 5 \mathrm{~A}-\mathrm{H} 5 \mathrm{~A}$ & 0.9300 \\
\hline C6- $-\mathrm{H} 6$ & 0.9300 & C6A-H6A & 0.9300 \\
\hline $\mathrm{C} 7-\mathrm{C} 26$ & $1.472(4)$ & $\mathrm{C} 7 \mathrm{~A}-\mathrm{C} 26 \mathrm{~A}$ & $1.502(4)$ \\
\hline $\mathrm{C} 7-\mathrm{C} 8$ & $1.497(4)$ & $\mathrm{C} 7 \mathrm{~A}-\mathrm{C} 8 \mathrm{~A}$ & $1.504(4)$ \\
\hline $\mathrm{C} 7-\mathrm{H} 7$ & 0.9800 & $\mathrm{C} 7 \mathrm{~A}-\mathrm{H} 7 \mathrm{~A}$ & 0.9800 \\
\hline $\mathrm{C} 8-\mathrm{C} 9$ & $1.506(3)$ & $\mathrm{C} 8 \mathrm{~A}-\mathrm{C} 9 \mathrm{~A}$ & $1.498(3)$ \\
\hline $\mathrm{C} 8-\mathrm{H} 8 \mathrm{~A}$ & 0.9700 & $\mathrm{C} 8 \mathrm{~A}-\mathrm{H} 8 \mathrm{~A} 1$ & 0.9700 \\
\hline $\mathrm{C} 8-\mathrm{H} 8 \mathrm{~B}$ & 0.9700 & $\mathrm{C} 8 \mathrm{~A}-\mathrm{H} 8 \mathrm{~A} 2$ & 0.9700 \\
\hline $\mathrm{C} 9-\mathrm{C} 24$ & $1.352(3)$ & $\mathrm{C} 9 \mathrm{~A}-\mathrm{C} 24 \mathrm{~A}$ & $1.355(3)$ \\
\hline $\mathrm{C} 9-\mathrm{C} 10$ & $1.463(3)$ & $\mathrm{C} 9 \mathrm{~A}-\mathrm{C} 10 \mathrm{~A}$ & $1.467(3)$ \\
\hline $\mathrm{C} 10-\mathrm{C} 11$ & $1.390(3)$ & $\mathrm{C} 10 \mathrm{~A}-\mathrm{C} 11 \mathrm{~A}$ & $1.383(3)$ \\
\hline $\mathrm{C} 10-\mathrm{C} 15$ & $1.406(3)$ & $\mathrm{C} 10 \mathrm{~A}-\mathrm{C} 15 \mathrm{~A}$ & $1.402(3)$ \\
\hline $\mathrm{C} 11-\mathrm{C} 12$ & $1.369(4)$ & $\mathrm{C} 11 \mathrm{~A}-\mathrm{C} 12 \mathrm{~A}$ & $1.369(4)$ \\
\hline C11-H11 & 0.9300 & $\mathrm{C} 11 \mathrm{~A}-\mathrm{H} 11 \mathrm{~A}$ & 0.9300 \\
\hline $\mathrm{C} 12-\mathrm{C} 13$ & $1.380(4)$ & $\mathrm{C} 12 \mathrm{~A}-\mathrm{C} 13 \mathrm{~A}$ & $1.377(4)$ \\
\hline $\mathrm{C} 12-\mathrm{H} 12$ & 0.9300 & $\mathrm{C} 12 \mathrm{~A}-\mathrm{H} 12 \mathrm{~A}$ & 0.9300 \\
\hline $\mathrm{C} 13-\mathrm{C} 14$ & $1.376(4)$ & $\mathrm{C} 13 \mathrm{~A}-\mathrm{C} 14 \mathrm{~A}$ & $1.362(4)$ \\
\hline $\mathrm{C} 14-\mathrm{C} 15$ & $1.368(4)$ & $\mathrm{C} 14 \mathrm{~A}-\mathrm{C} 15 \mathrm{~A}$ & $1.378(4)$ \\
\hline C14-H14 & 0.9300 & $\mathrm{C} 14 \mathrm{~A}-\mathrm{H} 14 \mathrm{~A}$ & 0.9300 \\
\hline C15-H15 & 0.9300 & $\mathrm{C} 15 \mathrm{~A}-\mathrm{H} 15 \mathrm{~A}$ & 0.9300 \\
\hline $\mathrm{C} 16-\mathrm{H} 16 \mathrm{~A}$ & 0.9600 & $\mathrm{C} 16 \mathrm{~A}-\mathrm{H} 16 \mathrm{D}$ & 0.9600 \\
\hline C16-H16B & 0.9600 & C16A-H16E & 0.9600 \\
\hline $\mathrm{C} 16-\mathrm{H} 16 \mathrm{C}$ & 0.9600 & $\mathrm{C} 16 \mathrm{~A}-\mathrm{H} 16 \mathrm{~F}$ & 0.9600 \\
\hline $\mathrm{C} 17-\mathrm{C} 18$ & $1.503(4)$ & $\mathrm{C} 17 \mathrm{~A}-\mathrm{C} 18 \mathrm{~A}$ & $1.488(4)$ \\
\hline C17-H17A & 0.9700 & $\mathrm{C} 17 \mathrm{~A}-\mathrm{H} 17 \mathrm{C}$ & 0.9700 \\
\hline C17-H17B & 0.9700 & $\mathrm{C} 17 \mathrm{~A}-\mathrm{H} 17 \mathrm{D}$ & 0.9700 \\
\hline $\mathrm{C} 18-\mathrm{C} 23$ & $1.362(4)$ & $\mathrm{C} 18 \mathrm{~A}-\mathrm{C} 23 \mathrm{~A}$ & $1.377(4)$ \\
\hline $\mathrm{C} 18-\mathrm{C} 19$ & $1.378(4)$ & $\mathrm{C} 18 \mathrm{~A}-\mathrm{C} 19 \mathrm{~A}$ & $1.379(4)$ \\
\hline $\mathrm{C} 19-\mathrm{C} 20$ & $1.380(4)$ & $\mathrm{C} 19 \mathrm{~A}-\mathrm{C} 20 \mathrm{~A}$ & $1.377(4)$ \\
\hline C19-H19 & 0.9300 & $\mathrm{C} 19 \mathrm{~A}-\mathrm{H} 19 \mathrm{~A}$ & 0.9300 \\
\hline $\mathrm{C} 20-\mathrm{C} 21$ & $1.344(5)$ & $\mathrm{C} 20 \mathrm{~A}-\mathrm{C} 21 \mathrm{~A}$ & $1.355(5)$ \\
\hline $\mathrm{C} 20-\mathrm{H} 20$ & 0.9300 & $\mathrm{C} 20 \mathrm{~A}-\mathrm{H} 20 \mathrm{~A}$ & 0.9300 \\
\hline $\mathrm{C} 21-\mathrm{C} 22$ & $1.345(5)$ & $\mathrm{C} 21 \mathrm{~A}-\mathrm{C} 22 \mathrm{~A}$ & $1.344(4)$ \\
\hline $\mathrm{C} 21-\mathrm{H} 21$ & 0.9300 & $\mathrm{C} 21 \mathrm{~A}-\mathrm{H} 21 \mathrm{~A}$ & 0.9300 \\
\hline $\mathrm{C} 22-\mathrm{C} 23$ & $1.367(4)$ & $\mathrm{C} 22 \mathrm{~A}-\mathrm{C} 23 \mathrm{~A}$ & $1.369(4)$ \\
\hline $\mathrm{C} 22-\mathrm{H} 22$ & 0.9300 & $\mathrm{C} 22 \mathrm{~A}-\mathrm{H} 22 \mathrm{~A}$ & 0.9300 \\
\hline $\mathrm{C} 23-\mathrm{H} 23$ & 0.9300 & $\mathrm{C} 23 \mathrm{~A}-\mathrm{H} 23 \mathrm{~A}$ & 0.9300 \\
\hline $\mathrm{C} 24-\mathrm{C} 25$ & $1.445(4)$ & $\mathrm{C} 24 \mathrm{~A}-\mathrm{C} 25 \mathrm{~A}$ & 1.449 (4) \\
\hline
\end{tabular}




\begin{tabular}{|c|c|c|c|}
\hline $\mathrm{C} 24-\mathrm{H} 24$ & 0.9300 & $\mathrm{C} 24 \mathrm{~A}-\mathrm{H} 24 \mathrm{~A}$ & 0.9300 \\
\hline $\mathrm{C} 25-\mathrm{C} 26$ & $1.510(4)$ & $\mathrm{C} 25 \mathrm{~A}-\mathrm{C} 26 \mathrm{~A}$ & $1.511(4)$ \\
\hline $\mathrm{C} 26-\mathrm{C} 27$ & $1.502(4)$ & $\mathrm{C} 26 \mathrm{~A}-\mathrm{C} 27 \mathrm{~A}$ & $1.507(4)$ \\
\hline $\mathrm{C} 26-\mathrm{H} 26$ & 0.9800 & $\mathrm{C} 26 \mathrm{~A}-\mathrm{H} 26 \mathrm{~A}$ & 0.9800 \\
\hline $\mathrm{C} 27-\mathrm{H} 27 \mathrm{~A}$ & 0.9600 & $\mathrm{C} 27 \mathrm{~A}-\mathrm{H} 27 \mathrm{D}$ & 0.9600 \\
\hline $\mathrm{C} 27-\mathrm{H} 27 \mathrm{~B}$ & 0.9600 & $\mathrm{C} 27 \mathrm{~A}-\mathrm{H} 27 \mathrm{E}$ & 0.9600 \\
\hline $\mathrm{C} 27-\mathrm{H} 27 \mathrm{C}$ & 0.9600 & $\mathrm{C} 27 \mathrm{~A}-\mathrm{H} 27 \mathrm{~F}$ & 0.9600 \\
\hline $\mathrm{C} 1-\mathrm{O} 2-\mathrm{C} 17$ & $117.8(2)$ & $\mathrm{C} 1 \mathrm{~A}-\mathrm{O} 2 \mathrm{~A}-\mathrm{C} 17 \mathrm{~A}$ & $118.6(2)$ \\
\hline $\mathrm{C} 13-\mathrm{O} 3-\mathrm{C} 16$ & $119.7(2)$ & $\mathrm{C} 13 \mathrm{~A}-\mathrm{O} 3 \mathrm{~A}-\mathrm{C} 16 \mathrm{~A}$ & $119.0(2)$ \\
\hline $\mathrm{C} 6-\mathrm{C} 1-\mathrm{C} 2$ & $119.9(3)$ & $\mathrm{C} 2 \mathrm{~A}-\mathrm{C} 1 \mathrm{~A}-\mathrm{C} 6 \mathrm{~A}$ & $119.6(3)$ \\
\hline $\mathrm{C} 6-\mathrm{C} 1-\mathrm{O} 2$ & $114.8(3)$ & $\mathrm{C} 2 \mathrm{~A}-\mathrm{C} 1 \mathrm{~A}-\mathrm{O} 2 \mathrm{~A}$ & $114.7(2)$ \\
\hline $\mathrm{C} 2-\mathrm{C} 1-\mathrm{O} 2$ & $125.3(3)$ & $\mathrm{C} 6 \mathrm{~A}-\mathrm{C} 1 \mathrm{~A}-\mathrm{O} 2 \mathrm{~A}$ & $125.7(3)$ \\
\hline $\mathrm{C} 1-\mathrm{C} 2-\mathrm{C} 3$ & $119.6(3)$ & $\mathrm{C} 1 \mathrm{~A}-\mathrm{C} 2 \mathrm{~A}-\mathrm{C} 3 \mathrm{~A}$ & $120.1(3)$ \\
\hline $\mathrm{C} 1-\mathrm{C} 2-\mathrm{H} 2$ & 120.2 & $\mathrm{C} 1 \mathrm{~A}-\mathrm{C} 2 \mathrm{~A}-\mathrm{H} 2 \mathrm{~A}$ & 120.0 \\
\hline $\mathrm{C} 3-\mathrm{C} 2-\mathrm{H} 2$ & 120.2 & $\mathrm{C} 3 \mathrm{~A}-\mathrm{C} 2 \mathrm{~A}-\mathrm{H} 2 \mathrm{~A}$ & 120.0 \\
\hline $\mathrm{C} 4-\mathrm{C} 3-\mathrm{C} 2$ & $121.7(3)$ & $\mathrm{C} 2 \mathrm{~A}-\mathrm{C} 3 \mathrm{~A}-\mathrm{C} 4 \mathrm{~A}$ & $121.4(3)$ \\
\hline $\mathrm{C} 4-\mathrm{C} 3-\mathrm{H} 3$ & 119.1 & $\mathrm{C} 2 \mathrm{~A}-\mathrm{C} 3 \mathrm{~A}-\mathrm{H} 3 \mathrm{~A}$ & 119.3 \\
\hline $\mathrm{C} 2-\mathrm{C} 3-\mathrm{H} 3$ & 119.1 & $\mathrm{C} 4 \mathrm{~A}-\mathrm{C} 3 \mathrm{~A}-\mathrm{H} 3 \mathrm{~A}$ & 119.3 \\
\hline $\mathrm{C} 3-\mathrm{C} 4-\mathrm{C} 5$ & $117.6(3)$ & $\mathrm{C} 5 \mathrm{~A}-\mathrm{C} 4 \mathrm{~A}-\mathrm{C} 3 \mathrm{~A}$ & $117.3(3)$ \\
\hline $\mathrm{C} 3-\mathrm{C} 4-\mathrm{C} 7$ & $122.0(3)$ & $\mathrm{C} 5 \mathrm{~A}-\mathrm{C} 4 \mathrm{~A}-\mathrm{C} 7 \mathrm{~A}$ & $121.6(3)$ \\
\hline $\mathrm{C} 5-\mathrm{C} 4-\mathrm{C} 7$ & $120.4(3)$ & $\mathrm{C} 3 \mathrm{~A}-\mathrm{C} 4 \mathrm{~A}-\mathrm{C} 7 \mathrm{~A}$ & $121.1(3)$ \\
\hline $\mathrm{C} 4-\mathrm{C} 5-\mathrm{C} 6$ & $122.2(3)$ & $\mathrm{C} 4 \mathrm{~A}-\mathrm{C} 5 \mathrm{~A}-\mathrm{C} 6 \mathrm{~A}$ & $122.2(3)$ \\
\hline $\mathrm{C} 4-\mathrm{C} 5-\mathrm{H} 5$ & 118.9 & $\mathrm{C} 4 \mathrm{~A}-\mathrm{C} 5 \mathrm{~A}-\mathrm{H} 5 \mathrm{~A}$ & 118.9 \\
\hline $\mathrm{C} 6-\mathrm{C} 5-\mathrm{H} 5$ & 118.9 & $\mathrm{C} 6 \mathrm{~A}-\mathrm{C} 5 \mathrm{~A}-\mathrm{H} 5 \mathrm{~A}$ & 118.9 \\
\hline $\mathrm{C} 1-\mathrm{C} 6-\mathrm{C} 5$ & $119.0(3)$ & $\mathrm{C} 1 \mathrm{~A}-\mathrm{C} 6 \mathrm{~A}-\mathrm{C} 5 \mathrm{~A}$ & $119.4(3)$ \\
\hline $\mathrm{C} 1-\mathrm{C} 6-\mathrm{H} 6$ & 120.5 & $\mathrm{C} 1 \mathrm{~A}-\mathrm{C} 6 \mathrm{~A}-\mathrm{H} 6 \mathrm{~A}$ & 120.3 \\
\hline $\mathrm{C} 5-\mathrm{C} 6-\mathrm{H} 6$ & 120.5 & $\mathrm{C} 5 \mathrm{~A}-\mathrm{C} 6 \mathrm{~A}-\mathrm{H} 6 \mathrm{~A}$ & 120.3 \\
\hline $\mathrm{C} 26-\mathrm{C} 7-\mathrm{C} 8$ & $113.8(3)$ & $\mathrm{C} 26 \mathrm{~A}-\mathrm{C} 7 \mathrm{~A}-\mathrm{C} 8 \mathrm{~A}$ & $113.1(2)$ \\
\hline $\mathrm{C} 26-\mathrm{C} 7-\mathrm{C} 4$ & $113.9(3)$ & $\mathrm{C} 26 \mathrm{~A}-\mathrm{C} 7 \mathrm{~A}-\mathrm{C} 4 \mathrm{~A}$ & $113.4(2)$ \\
\hline $\mathrm{C} 8-\mathrm{C} 7-\mathrm{C} 4$ & $112.2(2)$ & $\mathrm{C} 8 \mathrm{~A}-\mathrm{C} 7 \mathrm{~A}-\mathrm{C} 4 \mathrm{~A}$ & $113.2(2)$ \\
\hline $\mathrm{C} 26-\mathrm{C} 7-\mathrm{H} 7$ & 105.3 & $\mathrm{C} 26 \mathrm{~A}-\mathrm{C} 7 \mathrm{~A}-\mathrm{H} 7 \mathrm{~A}$ & 105.4 \\
\hline $\mathrm{C} 8-\mathrm{C} 7-\mathrm{H} 7$ & 105.3 & $\mathrm{C} 8 \mathrm{~A}-\mathrm{C} 7 \mathrm{~A}-\mathrm{H} 7 \mathrm{~A}$ & 105.4 \\
\hline $\mathrm{C} 4-\mathrm{C} 7-\mathrm{H} 7$ & 105.3 & $\mathrm{C} 4 \mathrm{~A}-\mathrm{C} 7 \mathrm{~A}-\mathrm{H} 7 \mathrm{~A}$ & 105.4 \\
\hline $\mathrm{C} 7-\mathrm{C} 8-\mathrm{C} 9$ & $115.0(2)$ & $\mathrm{C} 9 \mathrm{~A}-\mathrm{C} 8 \mathrm{~A}-\mathrm{C} 7 \mathrm{~A}$ & $114.1(2)$ \\
\hline $\mathrm{C} 7-\mathrm{C} 8-\mathrm{H} 8 \mathrm{~A}$ & 108.5 & $\mathrm{C} 9 \mathrm{~A}-\mathrm{C} 8 \mathrm{~A}-\mathrm{H} 8 \mathrm{~A} 1$ & 108.7 \\
\hline $\mathrm{C} 9-\mathrm{C} 8-\mathrm{H} 8 \mathrm{~A}$ & 108.5 & $\mathrm{C} 7 \mathrm{~A}-\mathrm{C} 8 \mathrm{~A}-\mathrm{H} 8 \mathrm{~A} 1$ & 108.7 \\
\hline $\mathrm{C} 7-\mathrm{C} 8-\mathrm{H} 8 \mathrm{~B}$ & 108.5 & $\mathrm{C} 9 \mathrm{~A}-\mathrm{C} 8 \mathrm{~A}-\mathrm{H} 8 \mathrm{~A} 2$ & 108.7 \\
\hline $\mathrm{C} 9-\mathrm{C} 8-\mathrm{H} 8 \mathrm{~B}$ & 108.5 & $\mathrm{C} 7 \mathrm{~A}-\mathrm{C} 8 \mathrm{~A}-\mathrm{H} 8 \mathrm{~A} 2$ & 108.7 \\
\hline $\mathrm{H} 8 \mathrm{~A}-\mathrm{C} 8-\mathrm{H} 8 \mathrm{~B}$ & 107.5 & $\mathrm{H} 8 \mathrm{~A} 1-\mathrm{C} 8 \mathrm{~A}-\mathrm{H} 8 \mathrm{~A} 2$ & 107.6 \\
\hline $\mathrm{C} 24-\mathrm{C} 9-\mathrm{C} 10$ & $122.4(2)$ & $\mathrm{C} 24 \mathrm{~A}-\mathrm{C} 9 \mathrm{~A}-\mathrm{C} 10 \mathrm{~A}$ & $122.3(2)$ \\
\hline $\mathrm{C} 24-\mathrm{C} 9-\mathrm{C} 8$ & $118.9(2)$ & $\mathrm{C} 24 \mathrm{~A}-\mathrm{C} 9 \mathrm{~A}-\mathrm{C} 8 \mathrm{~A}$ & $119.0(2)$ \\
\hline $\mathrm{C} 10-\mathrm{C} 9-\mathrm{C} 8$ & $118.7(2)$ & $\mathrm{C} 10 \mathrm{~A}-\mathrm{C} 9 \mathrm{~A}-\mathrm{C} 8 \mathrm{~A}$ & $118.7(2)$ \\
\hline $\mathrm{C} 11-\mathrm{C} 10-\mathrm{C} 15$ & $115.9(2)$ & $\mathrm{C} 11 \mathrm{~A}-\mathrm{C} 10 \mathrm{~A}-\mathrm{C} 15 \mathrm{~A}$ & $115.9(2)$ \\
\hline $\mathrm{C} 11-\mathrm{C} 10-\mathrm{C} 9$ & $122.9(2)$ & $\mathrm{C} 11 \mathrm{~A}-\mathrm{C} 10 \mathrm{~A}-\mathrm{C} 9 \mathrm{~A}$ & $122.4(2)$ \\
\hline $\mathrm{C} 15-\mathrm{C} 10-\mathrm{C} 9$ & $121.2(2)$ & $\mathrm{C} 15 \mathrm{~A}-\mathrm{C} 10 \mathrm{~A}-\mathrm{C} 9 \mathrm{~A}$ & $121.7(2)$ \\
\hline $\mathrm{C} 12-\mathrm{C} 11-\mathrm{C} 10$ & $122.5(3)$ & $\mathrm{C} 12 \mathrm{~A}-\mathrm{C} 11 \mathrm{~A}-\mathrm{C} 10 \mathrm{~A}$ & $123.2(2)$ \\
\hline $\mathrm{C} 12-\mathrm{C} 11-\mathrm{H} 11$ & 118.7 & $\mathrm{C} 12 \mathrm{~A}-\mathrm{C} 11 \mathrm{~A}-\mathrm{H} 11 \mathrm{~A}$ & 118.4 \\
\hline
\end{tabular}




\begin{tabular}{|c|c|c|c|}
\hline $\mathrm{C} 10-\mathrm{C} 11-\mathrm{H} 11$ & 118.7 & $\mathrm{C} 10 \mathrm{~A}-\mathrm{C} 11 \mathrm{~A}-\mathrm{H} 11 \mathrm{~A}$ & 118.4 \\
\hline $\mathrm{C} 11-\mathrm{C} 12-\mathrm{C} 13$ & $120.4(3)$ & $\mathrm{C} 11 \mathrm{~A}-\mathrm{C} 12 \mathrm{~A}-\mathrm{C} 13 \mathrm{~A}$ & $119.6(3)$ \\
\hline $\mathrm{C} 11-\mathrm{C} 12-\mathrm{H} 12$ & 119.8 & $\mathrm{C} 11 \mathrm{~A}-\mathrm{C} 12 \mathrm{~A}-\mathrm{H} 12 \mathrm{~A}$ & 120.2 \\
\hline $\mathrm{C} 13-\mathrm{C} 12-\mathrm{H} 12$ & 119.8 & $\mathrm{C} 13 \mathrm{~A}-\mathrm{C} 12 \mathrm{~A}-\mathrm{H} 12 \mathrm{~A}$ & 120.2 \\
\hline $\mathrm{O} 3-\mathrm{C} 13-\mathrm{C} 14$ & $116.1(3)$ & $\mathrm{O} 3 \mathrm{~A}-\mathrm{C} 13 \mathrm{~A}-\mathrm{C} 14 \mathrm{~A}$ & $116.2(3)$ \\
\hline $\mathrm{O} 3-\mathrm{C} 13-\mathrm{C} 12$ & $125.5(3)$ & $\mathrm{O} 3 \mathrm{~A}-\mathrm{C} 13 \mathrm{~A}-\mathrm{C} 12 \mathrm{~A}$ & $124.9(3)$ \\
\hline $\mathrm{C} 14-\mathrm{C} 13-\mathrm{C} 12$ & $118.4(3)$ & $\mathrm{C} 14 \mathrm{~A}-\mathrm{C} 13 \mathrm{~A}-\mathrm{C} 12 \mathrm{~A}$ & $118.9(3)$ \\
\hline $\mathrm{C} 15-\mathrm{C} 14-\mathrm{C} 13$ & $121.1(3)$ & $\mathrm{C} 13 \mathrm{~A}-\mathrm{C} 14 \mathrm{~A}-\mathrm{C} 15 \mathrm{~A}$ & $121.5(3)$ \\
\hline $\mathrm{C} 15-\mathrm{C} 14-\mathrm{H} 14$ & 119.4 & $\mathrm{C} 13 \mathrm{~A}-\mathrm{C} 14 \mathrm{~A}-\mathrm{H} 14 \mathrm{~A}$ & 119.3 \\
\hline $\mathrm{C} 13-\mathrm{C} 14-\mathrm{H} 14$ & 119.4 & $\mathrm{C} 15 \mathrm{~A}-\mathrm{C} 14 \mathrm{~A}-\mathrm{H} 14 \mathrm{~A}$ & 119.3 \\
\hline $\mathrm{C} 14-\mathrm{C} 15-\mathrm{C} 10$ & $121.5(3)$ & $\mathrm{C} 14 \mathrm{~A}-\mathrm{C} 15 \mathrm{~A}-\mathrm{C} 10 \mathrm{~A}$ & $120.8(3)$ \\
\hline $\mathrm{C} 14-\mathrm{C} 15-\mathrm{H} 15$ & 119.2 & $\mathrm{C} 14 \mathrm{~A}-\mathrm{C} 15 \mathrm{~A}-\mathrm{H} 15 \mathrm{~A}$ & 119.6 \\
\hline $\mathrm{C} 10-\mathrm{C} 15-\mathrm{H} 15$ & 119.2 & $\mathrm{C} 10 \mathrm{~A}-\mathrm{C} 15 \mathrm{~A}-\mathrm{H} 15 \mathrm{~A}$ & 119.6 \\
\hline $\mathrm{O} 3-\mathrm{C} 16-\mathrm{H} 16 \mathrm{~A}$ & 109.5 & $\mathrm{O} 3 \mathrm{~A}-\mathrm{C} 16 \mathrm{~A}-\mathrm{H} 16 \mathrm{D}$ & 109.5 \\
\hline $\mathrm{O} 3-\mathrm{C} 16-\mathrm{H} 16 \mathrm{~B}$ & 109.5 & $\mathrm{O} 3 \mathrm{~A}-\mathrm{C} 16 \mathrm{~A}-\mathrm{H} 16 \mathrm{E}$ & 109.5 \\
\hline $\mathrm{H} 16 \mathrm{~A}-\mathrm{C} 16-\mathrm{H} 16 \mathrm{~B}$ & 109.5 & $\mathrm{H} 16 \mathrm{D}-\mathrm{C} 16 \mathrm{~A}-\mathrm{H} 16 \mathrm{E}$ & 109.5 \\
\hline $\mathrm{O} 3-\mathrm{C} 16-\mathrm{H} 16 \mathrm{C}$ & 109.5 & $\mathrm{O} 3 \mathrm{~A}-\mathrm{C} 16 \mathrm{~A}-\mathrm{H} 16 \mathrm{~F}$ & 109.5 \\
\hline $\mathrm{H} 16 \mathrm{~A}-\mathrm{C} 16-\mathrm{H} 16 \mathrm{C}$ & 109.5 & $\mathrm{H} 16 \mathrm{D}-\mathrm{C} 16 \mathrm{~A}-\mathrm{H} 16 \mathrm{~F}$ & 109.5 \\
\hline $\mathrm{H} 16 \mathrm{~B}-\mathrm{C} 16-\mathrm{H} 16 \mathrm{C}$ & 109.5 & $\mathrm{H} 16 \mathrm{E}-\mathrm{C} 16 \mathrm{~A}-\mathrm{H} 16 \mathrm{~F}$ & 109.5 \\
\hline $\mathrm{O} 2-\mathrm{C} 17-\mathrm{C} 18$ & $108.1(3)$ & $\mathrm{O} 2 \mathrm{~A}-\mathrm{C} 17 \mathrm{~A}-\mathrm{C} 18 \mathrm{~A}$ & $108.7(2)$ \\
\hline $\mathrm{O} 2-\mathrm{C} 17-\mathrm{H} 17 \mathrm{~A}$ & 110.1 & $\mathrm{O} 2 \mathrm{~A}-\mathrm{C} 17 \mathrm{~A}-\mathrm{H} 17 \mathrm{C}$ & 110.0 \\
\hline $\mathrm{C} 18-\mathrm{C} 17-\mathrm{H} 17 \mathrm{~A}$ & 110.1 & $\mathrm{C} 18 \mathrm{~A}-\mathrm{C} 17 \mathrm{~A}-\mathrm{H} 17 \mathrm{C}$ & 110.0 \\
\hline $\mathrm{O} 2-\mathrm{C} 17-\mathrm{H} 17 \mathrm{~B}$ & 110.1 & $\mathrm{O} 2 \mathrm{~A}-\mathrm{C} 17 \mathrm{~A}-\mathrm{H} 17 \mathrm{D}$ & 110.0 \\
\hline $\mathrm{C} 18-\mathrm{C} 17-\mathrm{H} 17 \mathrm{~B}$ & 110.1 & $\mathrm{C} 18 \mathrm{~A}-\mathrm{C} 17 \mathrm{~A}-\mathrm{H} 17 \mathrm{D}$ & 110.0 \\
\hline $\mathrm{H} 17 \mathrm{~A}-\mathrm{C} 17-\mathrm{H} 17 \mathrm{~B}$ & 108.4 & $\mathrm{H} 17 \mathrm{C}-\mathrm{C} 17 \mathrm{~A}-\mathrm{H} 17 \mathrm{D}$ & 108.3 \\
\hline $\mathrm{C} 23-\mathrm{C} 18-\mathrm{C} 19$ & $117.8(3)$ & $\mathrm{C} 23 \mathrm{~A}-\mathrm{C} 18 \mathrm{~A}-\mathrm{C} 19 \mathrm{~A}$ & $117.3(3)$ \\
\hline $\mathrm{C} 23-\mathrm{C} 18-\mathrm{C} 17$ & $121.7(3)$ & $\mathrm{C} 23 \mathrm{~A}-\mathrm{C} 18 \mathrm{~A}-\mathrm{C} 17 \mathrm{~A}$ & $121.0(3)$ \\
\hline $\mathrm{C} 19-\mathrm{C} 18-\mathrm{C} 17$ & $120.5(3)$ & $\mathrm{C} 19 \mathrm{~A}-\mathrm{C} 18 \mathrm{~A}-\mathrm{C} 17 \mathrm{~A}$ & $121.5(3)$ \\
\hline $\mathrm{C} 18-\mathrm{C} 19-\mathrm{C} 20$ & $119.7(3)$ & $\mathrm{C} 20 \mathrm{~A}-\mathrm{C} 19 \mathrm{~A}-\mathrm{C} 18 \mathrm{~A}$ & $120.1(3)$ \\
\hline $\mathrm{C} 18-\mathrm{C} 19-\mathrm{H} 19$ & 120.2 & $\mathrm{C} 20 \mathrm{~A}-\mathrm{C} 19 \mathrm{~A}-\mathrm{H} 19 \mathrm{~A}$ & 119.9 \\
\hline $\mathrm{C} 20-\mathrm{C} 19-\mathrm{H} 19$ & 120.2 & $\mathrm{C} 18 \mathrm{~A}-\mathrm{C} 19 \mathrm{~A}-\mathrm{H} 19 \mathrm{~A}$ & 119.9 \\
\hline $\mathrm{C} 21-\mathrm{C} 20-\mathrm{C} 19$ & $121.3(3)$ & $\mathrm{C} 21 \mathrm{~A}-\mathrm{C} 20 \mathrm{~A}-\mathrm{C} 19 \mathrm{~A}$ & $121.5(3)$ \\
\hline $\mathrm{C} 21-\mathrm{C} 20-\mathrm{H} 20$ & 119.4 & $\mathrm{C} 21 \mathrm{~A}-\mathrm{C} 20 \mathrm{~A}-\mathrm{H} 20 \mathrm{~A}$ & 119.2 \\
\hline $\mathrm{C} 19-\mathrm{C} 20-\mathrm{H} 20$ & 119.4 & $\mathrm{C} 19 \mathrm{~A}-\mathrm{C} 20 \mathrm{~A}-\mathrm{H} 20 \mathrm{~A}$ & 119.2 \\
\hline $\mathrm{C} 20-\mathrm{C} 21-\mathrm{C} 22$ & $119.3(4)$ & $\mathrm{C} 22 \mathrm{~A}-\mathrm{C} 21 \mathrm{~A}-\mathrm{C} 20 \mathrm{~A}$ & $118.7(3)$ \\
\hline $\mathrm{C} 20-\mathrm{C} 21-\mathrm{H} 21$ & 120.4 & $\mathrm{C} 22 \mathrm{~A}-\mathrm{C} 21 \mathrm{~A}-\mathrm{H} 21 \mathrm{~A}$ & 120.7 \\
\hline $\mathrm{C} 22-\mathrm{C} 21-\mathrm{H} 21$ & 120.4 & $\mathrm{C} 20 \mathrm{~A}-\mathrm{C} 21 \mathrm{~A}-\mathrm{H} 21 \mathrm{~A}$ & 120.7 \\
\hline $\mathrm{C} 21-\mathrm{C} 22-\mathrm{C} 23$ & $120.6(4)$ & $\mathrm{C} 21 \mathrm{~A}-\mathrm{C} 22 \mathrm{~A}-\mathrm{C} 23 \mathrm{~A}$ & $121.2(3)$ \\
\hline $\mathrm{C} 21-\mathrm{C} 22-\mathrm{H} 22$ & 119.7 & $\mathrm{C} 21 \mathrm{~A}-\mathrm{C} 22 \mathrm{~A}-\mathrm{H} 22 \mathrm{~A}$ & 119.4 \\
\hline $\mathrm{C} 23-\mathrm{C} 22-\mathrm{H} 22$ & 119.7 & $\mathrm{C} 23 \mathrm{~A}-\mathrm{C} 22 \mathrm{~A}-\mathrm{H} 22 \mathrm{~A}$ & 119.4 \\
\hline $\mathrm{C} 18-\mathrm{C} 23-\mathrm{C} 22$ & $121.4(3)$ & $\mathrm{C} 22 \mathrm{~A}-\mathrm{C} 23 \mathrm{~A}-\mathrm{C} 18 \mathrm{~A}$ & $121.2(3)$ \\
\hline $\mathrm{C} 18-\mathrm{C} 23-\mathrm{H} 23$ & 119.3 & $\mathrm{C} 22 \mathrm{~A}-\mathrm{C} 23 \mathrm{~A}-\mathrm{H} 23 \mathrm{~A}$ & 119.4 \\
\hline $\mathrm{C} 22-\mathrm{C} 23-\mathrm{H} 23$ & 119.3 & $\mathrm{C} 18 \mathrm{~A}-\mathrm{C} 23 \mathrm{~A}-\mathrm{H} 23 \mathrm{~A}$ & 119.4 \\
\hline $\mathrm{C} 9-\mathrm{C} 24-\mathrm{C} 25$ & $123.6(3)$ & $\mathrm{C} 9 \mathrm{~A}-\mathrm{C} 24 \mathrm{~A}-\mathrm{C} 25 \mathrm{~A}$ & $124.0(2)$ \\
\hline C9-C24-H24 & 118.2 & $\mathrm{C} 9 \mathrm{~A}-\mathrm{C} 24 \mathrm{~A}-\mathrm{H} 24 \mathrm{~A}$ & 118.0 \\
\hline $\mathrm{C} 25-\mathrm{C} 24-\mathrm{H} 24$ & 118.2 & $\mathrm{C} 25 \mathrm{~A}-\mathrm{C} 24 \mathrm{~A}-\mathrm{H} 24 \mathrm{~A}$ & 118.0 \\
\hline $\mathrm{O} 1-\mathrm{C} 25-\mathrm{C} 24$ & $120.8(3)$ & $\mathrm{O} 1 \mathrm{~A}-\mathrm{C} 25 \mathrm{~A}-\mathrm{C} 24 \mathrm{~A}$ & $120.5(2)$ \\
\hline $\mathrm{O} 1-\mathrm{C} 25-\mathrm{C} 26$ & $120.9(3)$ & $\mathrm{O} 1 \mathrm{~A}-\mathrm{C} 25 \mathrm{~A}-\mathrm{C} 26 \mathrm{~A}$ & $121.3(3)$ \\
\hline
\end{tabular}




\begin{tabular}{|c|c|}
\hline $\mathrm{C} 24-\mathrm{C} 25-\mathrm{C} 26$ & $118.0(2)$ \\
\hline $\mathrm{C} 7-\mathrm{C} 26-\mathrm{C} 27$ & $117.1(3)$ \\
\hline $\mathrm{C} 7-\mathrm{C} 26-\mathrm{C} 25$ & $111.6(3)$ \\
\hline $\mathrm{C} 27-\mathrm{C} 26-\mathrm{C} 25$ & $112.6(2)$ \\
\hline $\mathrm{C} 7-\mathrm{C} 26-\mathrm{H} 26$ & 104.8 \\
\hline $\mathrm{C} 27-\mathrm{C} 26-\mathrm{H} 26$ & 104.8 \\
\hline $\mathrm{C} 25-\mathrm{C} 26-\mathrm{H} 26$ & 104.8 \\
\hline $\mathrm{C} 26-\mathrm{C} 27-\mathrm{H} 27 \mathrm{~A}$ & 109.5 \\
\hline $\mathrm{C} 26-\mathrm{C} 27-\mathrm{H} 27 \mathrm{~B}$ & 109.5 \\
\hline $\mathrm{H} 27 \mathrm{~A}-\mathrm{C} 27-\mathrm{H} 27 \mathrm{~B}$ & 109.5 \\
\hline $\mathrm{C} 26-\mathrm{C} 27-\mathrm{H} 27 \mathrm{C}$ & 109.5 \\
\hline $\mathrm{H} 27 \mathrm{~A}-\mathrm{C} 27-\mathrm{H} 27 \mathrm{C}$ & 109.5 \\
\hline $\mathrm{H} 27 \mathrm{~B}-\mathrm{C} 27-\mathrm{H} 27 \mathrm{C}$ & 109.5 \\
\hline $\mathrm{C} 17-\mathrm{O} 2-\mathrm{C} 1-\mathrm{C} 6$ & $179.5(2)$ \\
\hline $\mathrm{C} 17-\mathrm{O} 2-\mathrm{C} 1-\mathrm{C} 2$ & $-0.2(4)$ \\
\hline $\mathrm{C} 6-\mathrm{C} 1-\mathrm{C} 2-\mathrm{C} 3$ & $-0.2(4)$ \\
\hline $\mathrm{O} 2-\mathrm{C} 1-\mathrm{C} 2-\mathrm{C} 3$ & $179.5(2)$ \\
\hline $\mathrm{C} 1-\mathrm{C} 2-\mathrm{C} 3-\mathrm{C} 4$ & $0.6(4)$ \\
\hline $\mathrm{C} 2-\mathrm{C} 3-\mathrm{C} 4-\mathrm{C} 5$ & $-0.3(4)$ \\
\hline $\mathrm{C} 2-\mathrm{C} 3-\mathrm{C} 4-\mathrm{C} 7$ & $179.1(2)$ \\
\hline $\mathrm{C} 3-\mathrm{C} 4-\mathrm{C} 5-\mathrm{C} 6$ & $-0.3(4)$ \\
\hline $\mathrm{C} 7-\mathrm{C} 4-\mathrm{C} 5-\mathrm{C} 6$ & $-179.7(2)$ \\
\hline $\mathrm{C} 2-\mathrm{C} 1-\mathrm{C} 6-\mathrm{C} 5$ & $-0.4(4)$ \\
\hline $\mathrm{O} 2-\mathrm{C} 1-\mathrm{C} 6-\mathrm{C} 5$ & $179.9(2)$ \\
\hline $\mathrm{C} 4-\mathrm{C} 5-\mathrm{C} 6-\mathrm{C} 1$ & $0.7(4)$ \\
\hline $\mathrm{C} 3-\mathrm{C} 4-\mathrm{C} 7-\mathrm{C} 26$ & $118.8(3)$ \\
\hline $\mathrm{C} 5-\mathrm{C} 4-\mathrm{C} 7-\mathrm{C} 26$ & $-61.8(4)$ \\
\hline $\mathrm{C} 3-\mathrm{C} 4-\mathrm{C} 7-\mathrm{C} 8$ & $-110.1(3)$ \\
\hline $\mathrm{C} 5-\mathrm{C} 4-\mathrm{C} 7-\mathrm{C} 8$ & $69.2(4)$ \\
\hline $\mathrm{C} 26-\mathrm{C} 7-\mathrm{C} 8-\mathrm{C} 9$ & $-44.2(4)$ \\
\hline $\mathrm{C} 4-\mathrm{C} 7-\mathrm{C} 8-\mathrm{C} 9$ & $-175.3(2)$ \\
\hline $\mathrm{C} 7-\mathrm{C} 8-\mathrm{C} 9-\mathrm{C} 24$ & $16.6(4)$ \\
\hline $\mathrm{C} 7-\mathrm{C} 8-\mathrm{C} 9-\mathrm{C} 10$ & $-166.5(2)$ \\
\hline $\mathrm{C} 24-\mathrm{C} 9-\mathrm{C} 10-\mathrm{C} 11$ & $-16.0(4)$ \\
\hline $\mathrm{C} 8-\mathrm{C} 9-\mathrm{C} 10-\mathrm{C} 11$ & $167.3(2)$ \\
\hline $\mathrm{C} 24-\mathrm{C} 9-\mathrm{C} 10-\mathrm{C} 15$ & $161.8(3)$ \\
\hline $\mathrm{C} 8-\mathrm{C} 9-\mathrm{C} 10-\mathrm{C} 15$ & $-14.9(4)$ \\
\hline $\mathrm{C} 15-\mathrm{C} 10-\mathrm{C} 11-\mathrm{C} 12$ & $-2.1(4)$ \\
\hline $\mathrm{C} 9-\mathrm{C} 10-\mathrm{C} 11-\mathrm{C} 12$ & $175.9(3)$ \\
\hline $\mathrm{C} 10-\mathrm{C} 11-\mathrm{C} 12-\mathrm{C} 13$ & $0.3(4)$ \\
\hline $\mathrm{C} 16-\mathrm{O} 3-\mathrm{C} 13-\mathrm{C} 14$ & $-166.5(3)$ \\
\hline $\mathrm{C} 16-\mathrm{O} 3-\mathrm{C} 13-\mathrm{C} 12$ & $13.2(5)$ \\
\hline $\mathrm{C} 11-\mathrm{C} 12-\mathrm{C} 13-\mathrm{O} 3$ & $-178.0(3)$ \\
\hline $\mathrm{C} 11-\mathrm{C} 12-\mathrm{C} 13-\mathrm{C} 14$ & $1.7(4)$ \\
\hline $\mathrm{O} 3-\mathrm{C} 13-\mathrm{C} 14-\mathrm{C} 15$ & $178.0(3)$ \\
\hline $\mathrm{C} 12-\mathrm{C} 13-\mathrm{C} 14-\mathrm{C} 15$ & $-1.8(4)$ \\
\hline $\mathrm{C} 13-\mathrm{C} 14-\mathrm{C} 15-\mathrm{C} 10$ & $-0.1(4)$ \\
\hline
\end{tabular}

$\begin{array}{ll}\mathrm{C} 24 \mathrm{~A}-\mathrm{C} 25 \mathrm{~A}-\mathrm{C} 26 \mathrm{~A} & 118.1(2) \\ \mathrm{C} 7 \mathrm{~A}-\mathrm{C} 26 \mathrm{~A}-\mathrm{C} 27 \mathrm{~A} & 115.5(2) \\ \mathrm{C} 7 \mathrm{~A}-\mathrm{C} 26 \mathrm{~A}-\mathrm{C} 25 \mathrm{~A} & 110.6(2) \\ \mathrm{C} 27 \mathrm{~A}-\mathrm{C} 26 \mathrm{~A}-\mathrm{C} 25 \mathrm{~A} & 112.3(2) \\ \mathrm{C} 7 \mathrm{~A}-\mathrm{C} 26 \mathrm{~A}-\mathrm{H} 26 \mathrm{~A} & 105.9 \\ \mathrm{C} 27 \mathrm{~A}-\mathrm{C} 26 \mathrm{~A}-\mathrm{H} 26 \mathrm{~A} & 105.9 \\ \mathrm{C} 25 \mathrm{~A}-\mathrm{C} 26 \mathrm{~A}-\mathrm{H} 26 \mathrm{~A} & 105.9 \\ \mathrm{C} 26 \mathrm{~A}-\mathrm{C} 27 \mathrm{~A}-\mathrm{H} 27 \mathrm{D} & 109.5 \\ \mathrm{C} 26 \mathrm{~A}-\mathrm{C} 27 \mathrm{~A}-\mathrm{H} 27 \mathrm{E} & 109.5 \\ \mathrm{H} 27 \mathrm{D}-\mathrm{C} 27 \mathrm{~A}-\mathrm{H} 27 \mathrm{E} & 109.5 \\ \mathrm{C} 26 \mathrm{~A}-\mathrm{C} 27 \mathrm{~A}-\mathrm{H} 27 \mathrm{~F} & 109.5 \\ \mathrm{H} 27 \mathrm{D}-\mathrm{C} 27 \mathrm{~A}-\mathrm{H} 27 \mathrm{~F} & 109.5 \\ \mathrm{H} 27 \mathrm{E}-\mathrm{C} 27 \mathrm{~A}-\mathrm{H} 27 \mathrm{~F} & 109.5\end{array}$

$\mathrm{C} 17 \mathrm{~A}-\mathrm{O} 2 \mathrm{~A}-\mathrm{C} 1 \mathrm{~A}-\mathrm{C} 2 \mathrm{~A} \quad-179.5$ (2)

$\mathrm{C} 17 \mathrm{~A}-\mathrm{O} 2 \mathrm{~A}-\mathrm{C} 1 \mathrm{~A}-\mathrm{C} 6 \mathrm{~A} \quad 0.1$ (4)

$\mathrm{C} 6 \mathrm{~A}-\mathrm{C} 1 \mathrm{~A}-\mathrm{C} 2 \mathrm{~A}-\mathrm{C} 3 \mathrm{~A} \quad-0.4(4)$

$\mathrm{O} 2 \mathrm{~A}-\mathrm{C} 1 \mathrm{~A}-\mathrm{C} 2 \mathrm{~A}-\mathrm{C} 3 \mathrm{~A} \quad 179.2(2)$

$\mathrm{C} 1 \mathrm{~A}-\mathrm{C} 2 \mathrm{~A}-\mathrm{C} 3 \mathrm{~A}-\mathrm{C} 4 \mathrm{~A}-1.3(4)$

$\mathrm{C} 2 \mathrm{~A}-\mathrm{C} 3 \mathrm{~A}-\mathrm{C} 4 \mathrm{~A}-\mathrm{C} 5 \mathrm{~A} \quad 1.9$ (4)

$\mathrm{C} 2 \mathrm{~A}-\mathrm{C} 3 \mathrm{~A}-\mathrm{C} 4 \mathrm{~A}-\mathrm{C} 7 \mathrm{~A} \quad-177.6(2)$

$\mathrm{C} 3 \mathrm{~A}-\mathrm{C} 4 \mathrm{~A}-\mathrm{C} 5 \mathrm{~A}-\mathrm{C} 6 \mathrm{~A} \quad-0.7$ (4)

$\mathrm{C} 7 \mathrm{~A}-\mathrm{C} 4 \mathrm{~A}-\mathrm{C} 5 \mathrm{~A}-\mathrm{C} 6 \mathrm{~A} \quad 178.7$ (2)

$\mathrm{C} 2 \mathrm{~A}-\mathrm{C} 1 \mathrm{~A}-\mathrm{C} 6 \mathrm{~A}-\mathrm{C} 5 \mathrm{~A} \quad 1.5$ (4)

$\mathrm{O} 2 \mathrm{~A}-\mathrm{C} 1 \mathrm{~A}-\mathrm{C} 6 \mathrm{~A}-\mathrm{C} 5 \mathrm{~A} \quad-178.1$ (2)

$\mathrm{C} 4 \mathrm{~A}-\mathrm{C} 5 \mathrm{~A}-\mathrm{C} 6 \mathrm{~A}-\mathrm{C} 1 \mathrm{~A} \quad-0.9(4)$

$\mathrm{C} 5 \mathrm{~A}-\mathrm{C} 4 \mathrm{~A}-\mathrm{C} 7 \mathrm{~A}-\mathrm{C} 26 \mathrm{~A} \quad-118.0$ (3)

$\mathrm{C} 3 \mathrm{~A}-\mathrm{C} 4 \mathrm{~A}-\mathrm{C} 7 \mathrm{~A}-\mathrm{C} 26 \mathrm{~A}$

$\mathrm{C} 5 \mathrm{~A}-\mathrm{C} 4 \mathrm{~A}-\mathrm{C} 7 \mathrm{~A}-\mathrm{C} 8 \mathrm{~A} \quad 111.5(3)$

$\mathrm{C} 3 \mathrm{~A}-\mathrm{C} 4 \mathrm{~A}-\mathrm{C} 7 \mathrm{~A}-\mathrm{C} 8 \mathrm{~A} \quad-69.1$ (3)

$\mathrm{C} 26 \mathrm{~A}-\mathrm{C} 7 \mathrm{~A}-\mathrm{C} 8 \mathrm{~A}-\mathrm{C} 9 \mathrm{~A} \quad 47.7$ (3)

$\mathrm{C} 4 \mathrm{~A}-\mathrm{C} 7 \mathrm{~A}-\mathrm{C} 8 \mathrm{~A}-\mathrm{C} 9 \mathrm{~A} \quad 178.4(2)$

$\mathrm{C} 7 \mathrm{~A}-\mathrm{C} 8 \mathrm{~A}-\mathrm{C} 9 \mathrm{~A}-\mathrm{C} 24 \mathrm{~A} \quad-20.0(4)$

$\mathrm{C} 7 \mathrm{~A}-\mathrm{C} 8 \mathrm{~A}-\mathrm{C} 9 \mathrm{~A}-\mathrm{C} 10 \mathrm{~A} \quad 161.4(2)$

$\mathrm{C} 24 \mathrm{~A}-\mathrm{C} 9 \mathrm{~A}-\mathrm{C} 10 \mathrm{~A}-\mathrm{C} 11 \mathrm{~A} \quad 20.8(4)$

$\mathrm{C} 8 \mathrm{~A}-\mathrm{C} 9 \mathrm{~A}-\mathrm{C} 10 \mathrm{~A}-\mathrm{C} 11 \mathrm{~A}-160.6(2)$

$\mathrm{C} 24 \mathrm{~A}-\mathrm{C} 9 \mathrm{~A}-\mathrm{C} 10 \mathrm{~A}-\mathrm{C} 15 \mathrm{~A} \quad-156.7$ (3)

$\mathrm{C} 8 \mathrm{~A}-\mathrm{C} 9 \mathrm{~A}-\mathrm{C} 10 \mathrm{~A}-\mathrm{C} 15 \mathrm{~A} \quad 21.8$ (4)

$\mathrm{C} 15 \mathrm{~A}-\mathrm{C} 10 \mathrm{~A}-\mathrm{C} 11 \mathrm{~A}-\mathrm{C} 12 \mathrm{~A} \quad 0.8(4)$

$\mathrm{C} 9 \mathrm{~A}-\mathrm{C} 10 \mathrm{~A}-\mathrm{C} 11 \mathrm{~A}-\mathrm{C} 12 \mathrm{~A}-176.8$ (3)

$\mathrm{C} 10 \mathrm{~A}-\mathrm{C} 11 \mathrm{~A}-\mathrm{C} 12 \mathrm{~A}-\mathrm{C} 13 \mathrm{~A} \quad 1.7(4)$

$\mathrm{C} 16 \mathrm{~A}-\mathrm{O} 3 \mathrm{~A}-\mathrm{C} 13 \mathrm{~A}-\mathrm{C} 14 \mathrm{~A} \quad 173.7$ (3)

$\mathrm{C} 16 \mathrm{~A}-\mathrm{O} 3 \mathrm{~A}-\mathrm{C} 13 \mathrm{~A}-\mathrm{C} 12 \mathrm{~A}-7.3(5)$

$\mathrm{C} 11 \mathrm{~A}-\mathrm{C} 12 \mathrm{~A}-\mathrm{C} 13 \mathrm{~A}-\mathrm{O} 3 \mathrm{~A} \quad 178.2(3)$

$\mathrm{C} 11 \mathrm{~A}-\mathrm{C} 12 \mathrm{~A}-\mathrm{C} 13 \mathrm{~A}-\mathrm{C} 14 \mathrm{~A}-2.8$ (4)

$\mathrm{O} 3 \mathrm{~A}-\mathrm{C} 13 \mathrm{~A}-\mathrm{C} 14 \mathrm{~A}-\mathrm{C} 15 \mathrm{~A}-179.5$ (3)

$\mathrm{C} 12 \mathrm{~A}-\mathrm{C} 13 \mathrm{~A}-\mathrm{C} 14 \mathrm{~A}-\mathrm{C} 15 \mathrm{~A} \quad 1.4(5)$

$\mathrm{C} 13 \mathrm{~A}-\mathrm{C} 14 \mathrm{~A}-\mathrm{C} 15 \mathrm{~A}-\mathrm{C} 10 \mathrm{~A} \quad 1.3(5)$ 


$\begin{array}{ll}\mathrm{C} 11-\mathrm{C} 10-\mathrm{C} 15-\mathrm{C} 14 & 2.0(4) \\ \mathrm{C} 9-\mathrm{C} 10-\mathrm{C} 15-\mathrm{C} 14 & -176.0(3) \\ \mathrm{C} 1-\mathrm{O} 2-\mathrm{C} 17-\mathrm{C} 18 & -173.3(2) \\ \mathrm{O} 2-\mathrm{C} 17-\mathrm{C} 18-\mathrm{C} 23 & 51.4(4) \\ \mathrm{O} 2-\mathrm{C} 17-\mathrm{C} 18-\mathrm{C} 19 & -130.6(3) \\ \mathrm{C} 23-\mathrm{C} 18-\mathrm{C} 19-\mathrm{C} 20 & 1.1(5) \\ \mathrm{C} 17-\mathrm{C} 18-\mathrm{C} 19-\mathrm{C} 20 & -177.0(3) \\ \mathrm{C} 18-\mathrm{C} 19-\mathrm{C} 20-\mathrm{C} 21 & -0.6(6) \\ \mathrm{C} 19-\mathrm{C} 20-\mathrm{C} 21-\mathrm{C} 22 & -0.2(7) \\ \mathrm{C} 20-\mathrm{C} 21-\mathrm{C} 22-\mathrm{C} 23 & 0.5(7) \\ \mathrm{C} 19-\mathrm{C} 18-\mathrm{C} 23-\mathrm{C} 22 & -0.8(5) \\ \mathrm{C} 17-\mathrm{C} 18-\mathrm{C} 23-\mathrm{C} 22 & 177.2(3) \\ \mathrm{C} 21-\mathrm{C} 22-\mathrm{C} 23-\mathrm{C} 18 & 0.1(7) \\ \mathrm{C} 10-\mathrm{C} 9-\mathrm{C} 24-\mathrm{C} 25 & -173.4(2) \\ \mathrm{C} 8-\mathrm{C} 9-\mathrm{C} 24-\mathrm{C} 25 & 3.4(4) \\ \mathrm{C} 9-\mathrm{C} 24-\mathrm{C} 25-\mathrm{O} 1 & 177.9(3) \\ \mathrm{C} 9-\mathrm{C} 24-\mathrm{C} 25-\mathrm{C} 26 & 4.0(4) \\ \mathrm{C} 8-\mathrm{C} 7-\mathrm{C} 26-\mathrm{C} 27 & -178.1(3) \\ \mathrm{C} 4-\mathrm{C} 7-\mathrm{C} 26-\mathrm{C} 27 & -47.8(4) \\ \mathrm{C} 8-\mathrm{C} 7-\mathrm{C} 26-\mathrm{C} 25 & 50.2(4) \\ \mathrm{C} 4-\mathrm{C} 7-\mathrm{C} 26-\mathrm{C} 25 & -179.5(3) \\ \mathrm{O} 1-\mathrm{C} 25-\mathrm{C} 26-\mathrm{C} 7 & 155.3(3) \\ \mathrm{C} 24-\mathrm{C} 25-\mathrm{C} 26-\mathrm{C} 7 & -30.8(4) \\ \mathrm{O} 1-\mathrm{C} 25-\mathrm{C} 26-\mathrm{C} 27 & 21.3(5) \\ \mathrm{C} 24-\mathrm{C} 25-\mathrm{C} 26-\mathrm{C} 27 & -164.8(3) \\ \end{array}$

$\begin{array}{ll}\mathrm{C} 11 \mathrm{~A}-\mathrm{C} 10 \mathrm{~A}-\mathrm{C} 15 \mathrm{~A}-\mathrm{C} 14 \mathrm{~A} & -2.3(4) \\ \mathrm{C} 9 \mathrm{~A}-\mathrm{C} 10 \mathrm{~A}-\mathrm{C} 15 \mathrm{~A}-\mathrm{C} 14 \mathrm{~A} & 175.4(3) \\ \mathrm{C} 1 \mathrm{~A}-\mathrm{O} 2 \mathrm{~A}-\mathrm{C} 17 \mathrm{~A}-\mathrm{C} 18 \mathrm{~A} & 169.1(2) \\ \mathrm{O} 2 \mathrm{~A}-\mathrm{C} 17 \mathrm{~A}-\mathrm{C} 18 \mathrm{~A}-\mathrm{C} 23 \mathrm{~A} & -55.5(4) \\ \mathrm{O} 2 \mathrm{~A}-\mathrm{C} 17 \mathrm{~A}-\mathrm{C} 18 \mathrm{~A}-\mathrm{C} 19 \mathrm{~A} & 128.7(3) \\ \mathrm{C} 23 \mathrm{~A}-\mathrm{C} 18 \mathrm{~A}-\mathrm{C} 19 \mathrm{~A}-\mathrm{C} 20 \mathrm{~A} & 0.1(5) \\ \mathrm{C} 17 \mathrm{~A}-\mathrm{C} 18 \mathrm{~A}-\mathrm{C} 19 \mathrm{~A}-\mathrm{C} 20 \mathrm{~A} & 176.1(3) \\ \mathrm{C} 18 \mathrm{~A}-\mathrm{C} 19 \mathrm{~A}-\mathrm{C} 20 \mathrm{~A}-\mathrm{C} 21 \mathrm{~A} & -0.1(6) \\ \mathrm{C} 19 \mathrm{~A}-\mathrm{C} 20 \mathrm{~A}-\mathrm{C} 21 \mathrm{~A}-\mathrm{C} 22 \mathrm{~A} & 0.6(6) \\ \mathrm{C} 20 \mathrm{~A}-\mathrm{C} 21 \mathrm{~A}-\mathrm{C} 22 \mathrm{~A}-\mathrm{C} 23 \mathrm{~A} & -1.0(6) \\ \mathrm{C} 21 \mathrm{~A}-\mathrm{C} 22 \mathrm{~A}-\mathrm{C} 23 \mathrm{~A}-\mathrm{C} 18 \mathrm{~A} & 1.0(6) \\ \mathrm{C} 19 \mathrm{~A}-\mathrm{C} 18 \mathrm{~A}-\mathrm{C} 23 \mathrm{~A}-\mathrm{C} 22 \mathrm{~A} & -0.5(5) \\ \mathrm{C} 17 \mathrm{~A}-\mathrm{C} 18 \mathrm{~A}-\mathrm{C} 23 \mathrm{~A}-\mathrm{C} 22 \mathrm{~A} & -176.5(3) \\ \mathrm{C} 10 \mathrm{~A}-\mathrm{C} 9 \mathrm{~A}-\mathrm{C} 24 \mathrm{~A}-\mathrm{C} 25 \mathrm{~A} & 176.1(2) \\ \mathrm{C} 8 \mathrm{~A}-\mathrm{C} 9 \mathrm{~A}-\mathrm{C} 24 \mathrm{~A}-\mathrm{C} 25 \mathrm{~A} & -2.4(4) \\ \mathrm{C} 9 \mathrm{~A}-\mathrm{C} 24 \mathrm{~A}-\mathrm{C} 25 \mathrm{~A}-\mathrm{O} 1 \mathrm{~A} & -179.3(3) \\ \text { C9A-C24A-C25A-C26A } & -3.0(4) \\ \mathrm{C} 8 \mathrm{~A}-\mathrm{C} 7 \mathrm{~A}-\mathrm{C} 26 \mathrm{~A}-\mathrm{C} 27 \mathrm{~A} & 179.4(2) \\ \text { C4A-C7A-C26A-C27A } & 48.9(4) \\ \text { C8A-C7A-C26A-C25A } & -51.6(3) \\ \text { C4A-C7A-C26A-C25A } & 177.8(2) \\ \text { O1A-C25A-C26A-C7A } & -153.9(3) \\ \text { C24A-C25A-C26A-C7A } & 29.9(4) \\ \text { O1A-C25A-C26A-C27A } & -23.3(4) \\ \text { C24A-C25A-C26A-C27A } & 160.5(3) \\ & \end{array}$

Hydrogen-bond geometry $\left(\AA,{ }^{\circ}\right)$

$\mathrm{Cg} 3$ and $\mathrm{Cg} 8$ are the centroids of rings $\mathrm{C} 10-\mathrm{C} 15$ and $\mathrm{C} 10 \mathrm{~A}-\mathrm{C} 15 \mathrm{~A}$, respectively.

\begin{tabular}{lllll}
\hline$D-\mathrm{H}^{\cdots} \cdots A$ & $D-\mathrm{H}$ & $\mathrm{H} \cdots A$ & $D \cdots A$ & $D-\mathrm{H}^{\cdots} A A$ \\
\hline $\mathrm{C} 2 A-\mathrm{H} 2 A \cdots C g 3^{\mathrm{i}}$ & 0.93 & 2.87 & $3.707(3)$ & 150 \\
$\mathrm{C} 16 A-\mathrm{H} 16 E \cdots C g 8^{\mathrm{ii}}$ & 0.96 & 2.92 & $3.866(5)$ & 168 \\
\hline
\end{tabular}

Symmetry codes: (i) $x,-y+3 / 2, z+1 / 2$; (ii) $x,-y+3 / 2, z-1 / 2$. 